\title{
Tandem Reactions to Construct Heterocycles via Phosphine-Catalyzed Umpolung Addition and Intramolecular Conjugate Addition
}

\author{
Cheng Lu and Xiyan Lu* \\ State Key Laboratory of Organometallic Chemistry, Shanghai Institute of Organic Chemistry,
}

Chinese Academy of Sciences, 354 Fenglin Lu, Shanghai, 200032, China

xylu@pub.sioc.ac.cn

\section{Supporting Information}

Compounds $1 \mathbf{d}^{1}, \mathbf{1} \mathbf{e}^{2}, \mathbf{1} \mathbf{f}^{1}, \mathbf{2} \mathbf{a}^{3}, \mathbf{2} \mathbf{b}^{4}, \mathbf{2} \mathbf{c}^{5}, \mathbf{3} \mathbf{b}^{6}, \mathbf{3} \mathbf{c}^{7}, \mathbf{3} \mathbf{d}^{8}, \mathbf{3} \mathbf{e}^{9}, \mathbf{4} \mathbf{a}^{10}, \mathbf{4} \mathbf{f}^{11}$ were prepared by the methods described in the literature cited at the end of the supporting information.

Typical procedure for the phosphine-catalyzed tandem nucleophilic additions:

To a solution of 1a (56 mg, $0.5 \mathrm{mmol})$ and triphenylphosphine $(6.5 \mathrm{mg}, 0.025$ $\mathrm{mol})$ in toluene $(1.5 \mathrm{~mL})$ at $70^{\circ} \mathrm{C}$ under nitrogen was added a solution of $\mathbf{2 b}(41 \mathrm{mg}, 0.5$ mmol) in toluene $(1 \mathrm{~mL})$. After the reaction was completed as monitored by TLC, the solvent was removed under reduced pressure and the residue was purified by flash chromatography on silica gel (ethyl acetate:petroleum ether $=2: 1$ ) to give $\mathbf{5 b}$ as a yellow oil ( $81 \mathrm{mg}, 84 \%)$.

For the reaction of $\mathbf{1 b}$ or $\mathbf{1 c}$ with $\mathbf{2 b}$ (Table 1, entries 4 and 5), a solution of 1 equiv of $\mathbf{2 b}$ in toluene was added dropwise to the solution of 5 equiv of $\mathbf{1 b}$ or $\mathbf{1} \mathbf{c}$ and 0.05 equiv of triphenylphosphine in toluene at $70^{\circ} \mathrm{C}$ by a syringe over the indicated 
time. For the reaction of $\mathbf{1 d}$ or $\mathbf{1 e}$ with $\mathbf{3 b}, \mathbf{3 c}, \mathbf{3 e}$ or $\mathbf{4 f}$ (Table 1, entries 9, 11, 12 and 14), 1.1 equiv of alkynone in toluene or $\mathrm{CH}_{3} \mathrm{CN}$ was added dropwise to the solution of 1 equiv of bifunctional nucleophile and 0.2 equiv of triphenylphosphine in toluene or $\mathrm{CH}_{3} \mathrm{CN}$ at $80^{\circ} \mathrm{C}$ by a syringe over $12 \mathrm{hrs}$ and the mixture was heated for another $12 \mathrm{hrs}$.

\section{2-Ethoxycarbonylmethyl-3,5,6,7-tetrahydro-2H-benzofuran-4-one (5a)}

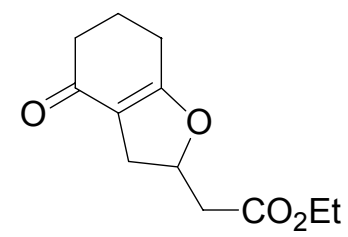

Oil. IR (neat) $v 2947,1736,1635,1404,1181 \mathrm{~cm}^{-1} .{ }^{1} \mathrm{H}$ NMR $\left(300 \mathrm{MHz}, \mathrm{CDCl}_{3}\right) \delta 5.14$ (m,1H), $4.14(\mathrm{q}, J=7.1 \mathrm{~Hz}, 2 \mathrm{H}), 2.98(\mathrm{dd}, J=14.6,10.1 \mathrm{~Hz}, 1 \mathrm{H}), 2.74(\mathrm{dd}, J=16,7.7$ $\mathrm{Hz}, 1 \mathrm{H}), 2.57(\mathrm{dd}, J=16,5.7 \mathrm{~Hz}, 1 \mathrm{H}), 2.45(\mathrm{dd}, J=14.6,5.7 \mathrm{~Hz}, 1 \mathrm{H}), 2.37$ ( t,$J=6.5$ $\mathrm{Hz}, 2 \mathrm{H}), 2.30(\mathrm{t}, J=6.5 \mathrm{~Hz}, 2 \mathrm{H}), 1.99(\mathrm{~m}, 2 \mathrm{H}), 1.23(\mathrm{t}, J=7.1 \mathrm{~Hz}, 3 \mathrm{H}) .{ }^{13} \mathrm{C} \mathrm{NMR}(75$ $\left.\mathrm{MHz}, \mathrm{CDCl}_{3}\right): \delta 195.25,176.71,169.53,112.48,80.91,60.61,40.53,36.09,31.10$ 23.57, 21.36, 13.88. MS (m/z) $224\left(\mathrm{M}^{+}\right)$, 179, 178, 150, 137 (100), 108, 81, 48, 41. Anal. Calcd for $\mathrm{C}_{12} \mathrm{H}_{16} \mathrm{O}_{4}$ : C, 64.27; H, 7.19. Found C, 64.18; H, 7.32.

\section{Cis-(3'-Ethoxycarbonylprop-2'-enyl)-2-hydroxycyclohex-1-en-6-one (6a)}<smiles>CCOC(=O)/C=C\CC1=C(O)CCCC1=O</smiles>

$\mathrm{Mp} 93-94^{\circ} \mathrm{C}$. IR (KBr) v 2956, 2558, 1722, 1645, 1565, 1359, 1272, 1190, 1076 $\mathrm{cm}^{-1}$. ${ }^{1} \mathrm{H}$ NMR $\left(300 \mathrm{MHz}, \mathrm{CDCl}_{3}\right) \delta 9.80(\mathrm{~s}, 1 \mathrm{H}), 6.29(\mathrm{dt}, J=11.3,9.2 \mathrm{~Hz}, 1 \mathrm{H}), 5.73(\mathrm{~d}, J=$ $11.3 \mathrm{~Hz}, 1 \mathrm{H}), 4.18(\mathrm{q}, J=7.1 \mathrm{~Hz}, 2 \mathrm{H}), 3.45(\mathrm{t}, J=9.2 \mathrm{~Hz}, 2 \mathrm{H}), 2.39$ (t, $J=6.5 \mathrm{~Hz}, 2 \mathrm{H})$, $2.29(\mathrm{t}, J=6.5 \mathrm{~Hz}, 2 \mathrm{H}), 1.86(\mathrm{~m}, 2 \mathrm{H}), 1.23(\mathrm{t}, J=7.1 \mathrm{~Hz}, 3 \mathrm{H}) .{ }^{13} \mathrm{C} \mathrm{NMR}(75 \mathrm{MHz}$, $\left.\mathrm{CDCl}_{3}\right): \delta 197.85,174.35,170.06,147.61,117.97,110.45,61.31,36.34,29.12,22.29$, 
20.51, 13.96. MS (m/z) $224\left(\mathrm{M}^{+}\right), 178,151,150(100), 149,137,55,43,41$. Anal.

Calcd for $\mathrm{C}_{12} \mathrm{H}_{16} \mathrm{O}_{4}$ : C, 64.27; H, 7.19. Found C, 64.10; H, 7.16.

Trans-(3'-Ethoxycarbonylprop-2'-enyl)-2-hydroxycyclohex-1-en-6-one (6b)<smiles>CCOC(=O)/C=C/CC1=C(O)CCCC1=O</smiles>

$\mathrm{Mp} 132-133^{\circ} \mathrm{C} . \mathrm{IR}(\mathrm{KBr}) v 2820,2606,1714,1567,1386,1273 \mathrm{~cm}^{-1} .{ }^{1} \mathrm{H}$ NMR $(300$ $\left.\mathrm{MHz}_{\mathrm{CDCl}}\right) \delta 10.07(\mathrm{~s}, 1 \mathrm{H}), 6.92(\mathrm{dt}, J=15.6,6.5 \mathrm{~Hz}, 1 \mathrm{H}), 5.77(\mathrm{~d}, J=15.6 \mathrm{~Hz}, 1 \mathrm{H})$, $4.14(\mathrm{q}, J=7.1 \mathrm{~Hz}, 2 \mathrm{H}), 3.19(\mathrm{t}, J=6.5 \mathrm{~Hz}, 1 \mathrm{H}), 2.47(\mathrm{~m}, 4 \mathrm{H}), 1.97(\mathrm{~m}, 2 \mathrm{H}), 1.25(\mathrm{t}, J$ $=7.1 \mathrm{~Hz}, 3 \mathrm{H}) .{ }^{13} \mathrm{C} \mathrm{NMR}\left(75 \mathrm{MHz}, \mathrm{CDCl}_{3}\right): \delta 167.52,147.53,120.60,111.93,60.30$, 32.70, 24.71, 20.63, 14.18. MS (m/z) $224\left(\mathrm{M}^{+}\right), 179,150(100), 149,95,55,43,42$. Anal. Calcd for $\mathrm{C}_{12} \mathrm{H}_{16} \mathrm{O}_{4}$ : C, 64.27; H, 7.19. Found C, 64.11; H, 7.13.

\section{2-Acetylmethyl-3,5,6,7-tetrahydro-2H-benzofuran-4-one (5b)}

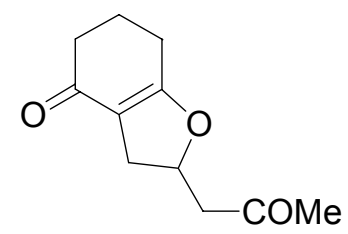

Oil. IR (neat) $v 2948,1715,1628,1406,1233,1181 \mathrm{~cm}^{-1} .{ }^{1} \mathrm{H}$ NMR $\left(300 \mathrm{MHz}, \mathrm{CDCl}_{3}\right)$ $\delta 5.21(\mathrm{~m}, 1 \mathrm{H}), 3.06(\mathrm{dd}, J=10.5,14.7 \mathrm{~Hz}, 1 \mathrm{H}), 2.98(\mathrm{dd}, J=17.4,7.2 \mathrm{~Hz}, 1 \mathrm{H}), 2.75$ (dd, $J=17.4,4.8 \mathrm{~Hz}, 1 \mathrm{H}), 2.46(\mathrm{dd}, J=14.7,7.2 \mathrm{~Hz}, 1 \mathrm{H}), 2.42(\mathrm{t}, J=6.2 \mathrm{~Hz}, 2 \mathrm{H}), 2.35$ $(\mathrm{t}, J=6.5 \mathrm{~Hz}, 2 \mathrm{H}), 2.22(\mathrm{~s}, 3 \mathrm{H}), 2.03(\mathrm{~m}, 2 \mathrm{H}) .{ }^{13} \mathrm{C} \mathrm{NMR}\left(75 \mathrm{MHz}, \mathrm{CDCl}_{3}\right): \delta 205.00$, 195.34, 176.84, 112.61, 80.56, 49.26, 36.17, 31.43, 30.36, 23.68, 21.43. MS (m/z) 194 $\left(\mathrm{M}^{+}\right), 137$ (100), 108, 96, 95, 81, 55, 43, 41. Anal. Calcd for $\mathrm{C}_{11} \mathrm{H}_{14} \mathrm{O}_{3}: \mathrm{C}, 68.02 ; \mathrm{H}$, 7.27. Found C, 64.04; H, 7.54. 
2-Benzoylmethyl-3,5,6,7-tetrahydro-2H-benzofuran-4-one (5c)

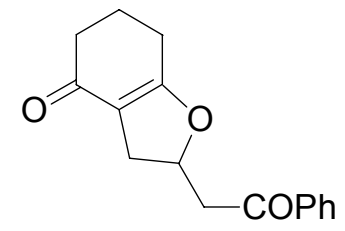

Mp 101-102. IR (KBr) v 2952, 1679, 1648, 1629, 1412, 1212, 1182, 758, $688 \mathrm{~cm}^{-1} .{ }^{1} \mathrm{H}$ NMR (300 MHz, $\left.\mathrm{CDCl}_{3}\right) \delta 7.96(\mathrm{~d}, J=7.2 \mathrm{~Hz}, 2 \mathrm{H}), 7.60(\mathrm{t}, J=7.2 \mathrm{~Hz}, 1 \mathrm{H}), 7.48(\mathrm{t}, J$ $=7.2 \mathrm{~Hz}), 5.43(\mathrm{~m}, 1 \mathrm{H}), 3.55(\mathrm{dd}, J=17.2,7.1 \mathrm{~Hz}), 3.25(\mathrm{dd}, J=17.2,6.8 \mathrm{~Hz}, 1 \mathrm{H})$, $3.13(\mathrm{dd}, J=14.6,7.2 \mathrm{~Hz}, 1 \mathrm{H}), 2.56(\mathrm{dd}, J=14.6,7.2 \mathrm{~Hz}, 1 \mathrm{H}), 2.40(\mathrm{t}, J=6.4 \mathrm{~Hz}, 2 \mathrm{H})$, $2.35(\mathrm{t}, J=6.4 \mathrm{~Hz}, 2 \mathrm{H}), 2.04(\mathrm{~m}, 2 \mathrm{H}) .{ }^{13} \mathrm{C} \mathrm{NMR}\left(75 \mathrm{MHz}, \mathrm{CDCl}_{3}\right): \delta$ 196.34, 195.33, $176.89,136.24,133.39,128.54,127.89,112.74,81.02,44.44,36.22,31.63,23.71$, 21.46. MS (m/z) $259\left(\mathrm{M}^{+}+1,100\right), 258\left(\mathrm{M}^{+}\right), 137,120,108,105,77,51$. Anal. Calcd for $\mathrm{C}_{16} \mathrm{H}_{16} \mathrm{O}_{3}: \mathrm{C}, 74.98 ; \mathrm{H}, 6.29$. Found C, 74.99; H, 6.31.

\section{2-Acetylmethyl-4-acetyl-5-methyl-2,3-dihydrofuran (5d)}

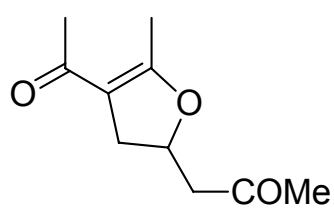

Oil. IR (neat) $v 2926,1716,1670,1618,1602,1324,1228 \mathrm{~cm}^{-1} .{ }^{1} \mathrm{H}$ NMR (300 MHz, $\left.\mathrm{CDCl}_{3}\right) \delta 5.02(\mathrm{~m}, 1 \mathrm{H}), 3.17(\mathrm{dd}, J=11.5,14.3 \mathrm{~Hz}, 1 \mathrm{H}), 2.96(\mathrm{dd}, J=17.0,7.1 \mathrm{~Hz}$, 1H), $2.71(\mathrm{dd}, J=17.0,6.2 \mathrm{~Hz}, 1 \mathrm{H}), 2.57$ (dd, $J=14.3,7.5 \mathrm{~Hz}, 1 \mathrm{H}), 2.21$ (s, 3H), 2.19 (s, 3H), $2.17(\mathrm{~s}, 3 \mathrm{H}) .{ }^{13} \mathrm{C}$ NMR (75 MHz, $\left.\mathrm{CDCl}_{3}\right): \delta 205.40,194.29,166.93,111.61$, 77.41, 49.25, 35.95, 30.40, 29.22, 14.73. MS (m/z) $182\left(\mathrm{M}^{+}\right), 125,109,97,43(100)$, 41. Anal. Calcd for $\mathrm{C}_{10} \mathrm{H}_{14} \mathrm{O}_{3}$ : C, 68.02; H, 7.27. Found C, 64.04; H, 7.54. 


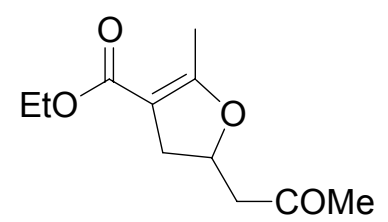

Mp 43-44 ${ }^{\circ} \mathrm{C}$. IR (KBr) v 2982, 1716, 1698, 1648, 1602, 1375, 1227, $1068 \mathrm{~cm}^{-1} .{ }^{1} \mathrm{H}$ NMR (300 MHz, $\left.\mathrm{CDCl}_{3}\right) \delta 5.04(\mathrm{~m}, 1 \mathrm{H}), 4.18(\mathrm{q}, J=7.2 \mathrm{~Hz}, 2 \mathrm{H}), 3.12(\mathrm{dd}, J=10.2$, 14.4 Hz. 1H), $2.96(\mathrm{dd}, J=17.1,7.0 \mathrm{~Hz}, 1 \mathrm{H}), 2.71(\mathrm{dd}, J=17.1,6.0 \mathrm{~Hz}, 1 \mathrm{H}), 2.51(\mathrm{dd}$, $J=14.4,7.5 \mathrm{~Hz}, 1 \mathrm{H}), 2.2(\mathrm{~s} 5,3 \mathrm{H}) 25,109,2.18(\mathrm{~s}, 3 \mathrm{H}), 1.29(\mathrm{t}, J=7.2 \mathrm{~Hz}, 3 \mathrm{H}) .{ }^{13} \mathrm{C}$ NMR (75 MHz, $\left.\mathrm{CDCl}_{3}\right): \delta 205.57,167.17,165.90,101.52,77.37,59.32,49.40,35.30$, 30.48, 14.27, 13.89. MS (m/z) $212\left(\mathrm{M}^{+}\right), 167,155,154,126,1102,43$ (100). Anal. Calcd for $\mathrm{C}_{11} \mathrm{H}_{16} \mathrm{O}_{4}: \mathrm{C}, 62.25 ; \mathrm{H}, 7.60$. Found $\mathrm{C}, 62.48 ; \mathrm{H}, 7.74$.

\section{2-Benzyloxycarbonylmethyl-3,5,6,7-tetrahydro-2H-benzofuran-4-one (5f)}

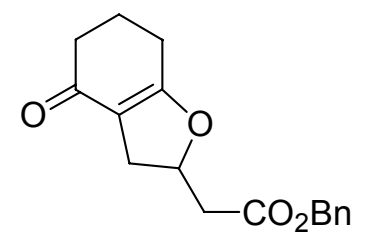

Oil. IR (neat) $v 2948,1737,1635,1404,1181,752,699 \mathrm{~cm}^{-1} .{ }^{1} \mathrm{H}$ NMR $(300 \mathrm{MHz}$, $\left.\mathrm{CDCl}_{3}\right) \delta 7.26-7.38(\mathrm{~m}, 5 \mathrm{H}), 5.21(\mathrm{~m}, 1 \mathrm{H}), 5.16(\mathrm{~S}, 2 \mathrm{H}), 3.02(\mathrm{dd}, J=10.2,14.7 \mathrm{~Hz}$ 1H), $2.82(\mathrm{dd}, J=16.2,7.8 \mathrm{~Hz}, 1 \mathrm{H}), 2.69(\mathrm{dd}, J=16.2,5.7 \mathrm{~Hz}, 1 \mathrm{H}), 2.51(\mathrm{dd}, J=14.7$, $7.2 \mathrm{~Hz}, 1 \mathrm{H}), 2.39(\mathrm{t}, J=6.3 \mathrm{~Hz}, 2 \mathrm{H}), 2.33(\mathrm{t}, J=6.3 \mathrm{~Hz}, 2 \mathrm{H}), 2.02(\mathrm{~m}, 2 \mathrm{H}) .{ }^{13} \mathrm{C} \mathrm{NMR}$ $\left(75 \mathrm{MHz}, \mathrm{CDCl}_{3}\right): \delta 195.38,176.77,169.55,135.33,128.50,128.30,128.18,112.66$, 80.93, 66.60, 40.68, 36.26, 31.30, 23.71, 21.49. MS (m/z) $210\left(\mathrm{M}^{+}\right), 178,150,137$ (100), 108, 43, 41. Anal. Calcd for $\mathrm{C}_{17} \mathrm{H}_{18} \mathrm{O}_{4}$ : C, 71.31; H, 6.34. Found C, 71.60; H, 6.73 . 


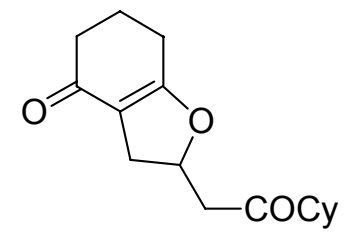

Oil. IR (neat) $v 2932,2856,1707,1648,1633,1404,1231,1182 \mathrm{~cm}^{-1} .{ }^{1} \mathrm{H}$ NMR $(300$ $\left.\mathrm{MHz}, \mathrm{CDCl}_{3}\right) \delta 5.22(\mathrm{~m}, 1 \mathrm{H}), 2.96-3.08(\mathrm{~m}, 2 \mathrm{H}), 2.74(\mathrm{dd}, J=17.1,5.8 \mathrm{~Hz}, 1 \mathrm{H})$, 2.32-2.45 (m, 5H), $2.03(\mathrm{~m}, 2 \mathrm{H}), 1.67-1.88(\mathrm{~m}, 5 \mathrm{H}), 1.18-1.40(\mathrm{~m}, 6 \mathrm{H}) .{ }^{13} \mathrm{C} \mathrm{NMR}(75$ $\left.\mathrm{MHz}, \mathrm{CDCl}_{3}\right): \delta 210.51,195.81,177.36,112.84,81.15,51.09,46.40,36.24,31.64$ 28.12, 28.04, 25.67, 25.46, 25.43, 23.88, 21.56. MS (m/z) $264\left(\mathrm{M}^{+}+2,100\right), 153,137$, 83, 81, 55, 41. Anal. Calcd for $\mathrm{C}_{16} \mathrm{H}_{22} \mathrm{O}_{3}$ : C, 73.25; H, 8.45. Found C, 72.96; H, 8.05.

\section{$N, N$ 'Di-(p-toluenesulfonyl)-2-acetylmethyl-piperazine (5h)}

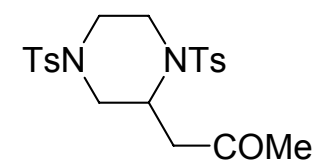

Mp 146-147 ${ }^{\circ} \mathrm{C} . \mathrm{IR}(\mathrm{KBr}) v 2924,1701,1341,1168,942,667 \mathrm{~cm}^{-1} .{ }^{1} \mathrm{H}$ NMR (300 MHz, $\left.\mathrm{CDCl}_{3}\right) \delta 7.60(\mathrm{~d}, J=9.0 \mathrm{~Hz}, 2 \mathrm{H}), 7.54(\mathrm{~d}, J=8.4 \mathrm{~Hz}, 2 \mathrm{H}), 7.41(\mathrm{~d}, J=8.4 \mathrm{~Hz}, 2 \mathrm{H})$, $7.25(\mathrm{~d}, J=9.0 \mathrm{~Hz}, 2 \mathrm{H}), 4.50(\mathrm{brd}, J=8.7 \mathrm{~Hz}, 1 \mathrm{H}), 3.80(\mathrm{dd}, J=13.2,1.5 \mathrm{~Hz}, 1 \mathrm{H})$, $3.69(\mathrm{dt}, J=11.7,1.5 \mathrm{~Hz}, 1 \mathrm{H}), 3.50(\mathrm{dt}, J=11.7,1.8 \mathrm{~Hz}, 1 \mathrm{H}), 3.07-3.20(\mathrm{~m}, 2 \mathrm{H}), 2.45$ $(\mathrm{s}, 3 \mathrm{H}), 2.41(\mathrm{~s}, 3 \mathrm{H}), 2.21-2.38(\mathrm{~m}, 3 \mathrm{H}), 2.09(\mathrm{~s}, 3 \mathrm{H}) .{ }^{13} \mathrm{C} \mathrm{NMR}\left(75 \mathrm{MHz}, \mathrm{CDCl}_{3}\right): \delta$ 205.21, 143.93, 143.72, 136.63, 132.21, 129.73, 129.66, 127.29, 126.81, 48.64, 47.97, 45.30, 41.28, 40.61, 30.28, 21.37, 21.32. MS (m/z) $392\left(\mathrm{M}^{+}-\mathrm{CH}_{3} \mathrm{COCH}_{3}\right), 295,253$, 155, 140, 91(100), 65. Anal. Calcd for $\mathrm{C}_{21} \mathrm{H}_{26} \mathrm{~N}_{2} \mathrm{O}_{5} \mathrm{~S}_{2}$ : C, 55.98; H, 5.82; N, 6.22. Found C, 55.99; H, 5.79; N, 6.05. 


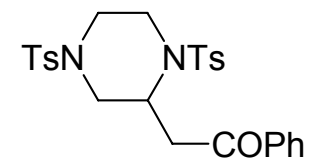

Workup up procedure: This product precipitated from the reaction mixture and was collected by filtration. Ether was added to the filtrate and the mixture was filtered again. The combined solid was washed with ether and crystallized from $\mathrm{CH}_{2} \mathrm{Cl}_{2}$-petroleum ether. (The crude product was almost pure when judged from its ${ }^{1} \mathrm{H}$ NMR.)

$\mathrm{Mp} 210-211^{\circ} \mathrm{C} . \mathrm{IR}(\mathrm{KBr}) v 2922,1682,1341,1170,938,670 \mathrm{~cm}^{-1} .{ }^{1} \mathrm{H}$ NMR $(300 \mathrm{MHz}$, $\left.\mathrm{CDCl}_{3}\right) \delta 7.59(\mathrm{~d}, J=8.4 \mathrm{~Hz}, 2 \mathrm{H}), 7.53-7.61(\mathrm{~m}, 5 \mathrm{H}), 7.44(\mathrm{t}, J=7.8 \mathrm{~Hz}, 2 \mathrm{H}), 7.28(\mathrm{~d}$, $J=8.4 \mathrm{~Hz}, 2 \mathrm{H}), 7.17(\mathrm{~d}, J=7.8 \mathrm{~Hz}, 2 \mathrm{H}), 4.73(\mathrm{brd}, J=8.7 \mathrm{~Hz}, 1 \mathrm{H}), 3.88(\mathrm{~d}, J=13.2$ $\mathrm{Hz}, 1 \mathrm{H}), 3.74(\mathrm{~d}, J=11.7,1 \mathrm{H}), 3.52-3.65(\mathrm{~m}, 2 \mathrm{H}), 3.21(\mathrm{dt}, J=3.0,13.2 \mathrm{~Hz}, 1 \mathrm{H}), 2.73$ $(\mathrm{d}, J=13.2 \mathrm{~Hz}, 2 \mathrm{H}), 2.42(\mathrm{~s}, 3 \mathrm{H}), 2.35-2.46(\mathrm{~m}, 2 \mathrm{H}), 2.32(\mathrm{~s}, 3 \mathrm{H}) .{ }^{13} \mathrm{C} \mathrm{NMR}(75 \mathrm{MHz}$, $\left.\mathrm{CDCl}_{3}\right): \delta 196.64,144.10,143.82,136.75,136.27,133.48,132.41,129.89,128.57$ 127.97, 127.55, 127.01, 49.30, 48.64, 45.67, 40.96, 36.67, 21.54, 21.43. MS (m/z) 407 $\left(\mathrm{M}^{+}\right.$-PhCO), 393, 392, 357, 237, 105 (100). Anal. Calcd for $\mathrm{C}_{26} \mathrm{H}_{28} \mathrm{~N}_{2} \mathrm{O}_{5} \mathrm{~S}_{2}: \mathrm{C}, 60.92 ; \mathrm{H}$, 5.51; N, 5.46. Found C, 60.95; H, 5.35; N, 5.66.

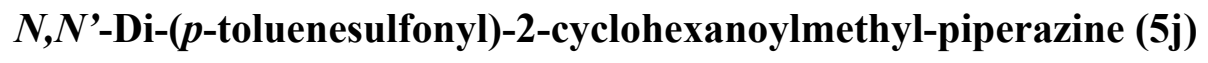

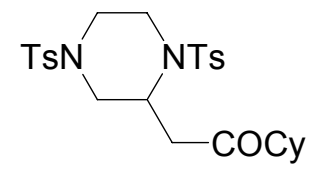

Mp 142-143으 IR (KBr) v 2931, 2855, 1702, 1344, 1162, $941 \mathrm{~cm}-1.1 \mathrm{H}$ NMR (300 $\left.\mathrm{MHz}, \mathrm{CDCl}_{3}\right) \delta 7.59(\mathrm{~d}, J=8.1 \mathrm{~Hz}, 2 \mathrm{H}), 7.53(\mathrm{~d}, J=8.1 \mathrm{~Hz}, 2 \mathrm{H}), 7.31(\mathrm{~d}, J=8.4 \mathrm{~Hz}$, 2H), $7.24(\mathrm{~d}, J=8.4 \mathrm{~Hz}, 2 \mathrm{H}), 4.51(\mathrm{brd}, J=8.4 \mathrm{~Hz}, 1 \mathrm{H}), 3.82(\mathrm{~d}, J=12.9 \mathrm{~Hz}, 1 \mathrm{H})$, $3.69(\mathrm{~d}, J=11.7,1 \mathrm{H}), 3.45(\mathrm{~d}, J=11.7 \mathrm{~Hz}, 1 \mathrm{H}), 3.18(\mathrm{dd}, J=10.5,18 \mathrm{~Hz}, 1 \mathrm{H}), 3.12$ 
(dt $J=3.3,12.9 \mathrm{~Hz}, 1 \mathrm{H}), 2.44(\mathrm{~s}, 3 \mathrm{H}), 2.40(\mathrm{~s}, 3 \mathrm{H}), 2.12-2.31(\mathrm{~m}, 4 \mathrm{H}), 1.63-1.77$ (m, 5H), 1.18-1.27(m, 5H). ${ }^{13} \mathrm{C}$ NMR (75 MHz, $\left.\mathrm{CDCl}_{3}\right): \delta 210.59,143.97,143.69,136.76$, $132.16,129.77,127.38,126.88,50.82,48.79,47.99,45.53,40.69,38.23,28.11,28.06$, 25.59, 25.37, 25.36, 21.45, 21.38. MS (m/z) $363\left(\mathrm{M}^{+}-\mathrm{Ts}\right), 253,237,208$ (100), 154, 91, 83, 55. Anal. Calcd for $\mathrm{C}_{26} \mathrm{H}_{34} \mathrm{~N}_{2} \mathrm{O}_{5} \mathrm{~S}_{2}$ : C, 60.21; H, 6.61; N, 5.40. Found C, 60.17; H, $6.49 ; \mathrm{N}, 5.19$.

$N$-(p-Toluenesulfonyl)-2-acetylmethylmorpholine (5k)

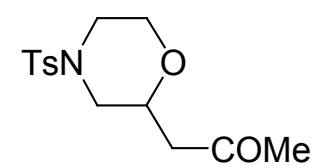

$\mathrm{Mp} 86-87^{\circ} \mathrm{C} \quad \mathrm{IR}(\mathrm{KBr}) v 2922,2899,1722$ 1344, 1160, $752 \mathrm{~cm}^{-1} .{ }^{1} \mathrm{H}$ NMR (300 MHz, $\left.\mathrm{CDCl}_{3}\right) \delta 7.63(\mathrm{~d}, J=8.1 \mathrm{~Hz}, 2 \mathrm{H}), 7.35(\mathrm{~d}, J=8.1 \mathrm{~Hz}, 2 \mathrm{H}), 4.02(\mathrm{~m}, 1 \mathrm{H}), 3.87(\mathrm{dq}, J=$ 1.7, $11.7 \mathrm{~Hz}, 1 \mathrm{H}), 3.68(\mathrm{dt}, J=2.7,11.7,1 \mathrm{H}), 3.53(\mathrm{~m}, 2 \mathrm{H}), 2.59(\mathrm{dd}, J=7.6,18.0 \mathrm{~Hz}$, 1H), $2.45(\mathrm{~s}, 3 \mathrm{H}), 2.39-2.48(\mathrm{~m}, 2 \mathrm{H}), 2.16(\mathrm{~s}, 3 \mathrm{H}), 2.15(\mathrm{~m}, 1 \mathrm{H}) .{ }^{13} \mathrm{C}$ NMR $(75 \mathrm{MHz}$ $\left.\mathrm{CDCl}_{3}\right): \delta 205.14,143.85,131.90,129.64,127.61,71.15,65.59,49.54,46.33,45.12$, 30.55, 21.36. MS (m/z) 407 ( $\left.\mathrm{M}^{+}-\mathrm{PhCO}\right), 393,392,357,237,105$ (100). Anal. Calcd for $\mathrm{C}_{14} \mathrm{H}_{19} \mathrm{NO}_{4} \mathrm{~S}: \mathrm{C}, 56.55 ; \mathrm{H}, 6.44 ; \mathrm{N}, 4.71$. Found $\mathrm{C}, 56.40 ; \mathrm{H}, 6.42 ; \mathrm{N}, 4.57$.

$N$-(p-Toluenesulfonyl)-2-cyclohexanoylmethylmorpholine (5l)

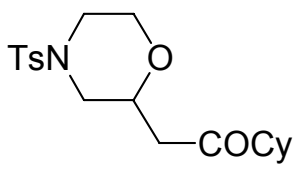

$\operatorname{Mp} 99-100^{\circ} \mathrm{C} \quad \mathrm{IR}(\mathrm{KBr}) v 2931,2854,17121348,1168,979,756 \mathrm{~cm}^{-1} .{ }^{1} \mathrm{H}$ NMR $(300$ $\left.\mathrm{MHz}, \mathrm{CDCl}_{3}\right) \delta 7.64(\mathrm{~d}, J=8.1 \mathrm{~Hz}, 2 \mathrm{H}), 7.36(\mathrm{~d}, J=8.1 \mathrm{~Hz}, 2 \mathrm{H}), 4.03(\mathrm{~m}, 1 \mathrm{H}), 3.86$ (dt, $J=10.2,1.5 \mathrm{~Hz}, 1 \mathrm{H}), 3.66(\mathrm{dt}, J=2.7,11.4,1 \mathrm{H}), 3.58(\mathrm{~d}, J=11.4 \mathrm{~Hz}, 1 \mathrm{H}), 3.49$ (d, $J=11.4 \mathrm{~Hz}, 1 \mathrm{H}), 2.66(\mathrm{dd}, J=7.5,16.5 \mathrm{~Hz}, 1 \mathrm{H}), 2.45(\mathrm{~s}, 3 \mathrm{H}), 2.40-2.49$ (m, 2H), 2.16 
(s, 3H), $2.31(\mathrm{~m}, 1 \mathrm{H}), 2.17(\mathrm{t}, J=10.7 \mathrm{~Hz}), 1.76-1.83(\mathrm{~m}, 4 \mathrm{H}), 1.66(\mathrm{~m}, 1 \mathrm{H}), 1.16-1.36$ (m, 5H). ${ }^{13} \mathrm{C} \mathrm{NMR}\left(75 \mathrm{MHz}, \mathrm{CDCl}_{3}\right): \delta 210.08,143.77,131.99,129.59,127.61,71.14$, $65.52,50.99,49.63,45.15,43.33,27.91,27.87,25.59,25.35,25.31,21.36 . \mathrm{MS}(\mathrm{m} / \mathrm{z})$ $366\left(\mathrm{M}^{+}+1\right), 254,239$ (100), 210, 91, 81, 55, 41. Anal. Calcd for $\mathrm{C}_{19} \mathrm{H}_{27} \mathrm{NO}_{4} \mathrm{~S}: \mathrm{C}$, 62.44; H, 3.83; N, 4.71. Found C, 62.45; H, 7.23; N, 3.71.

Crystallgraphic data for 5l: $\mathrm{C}_{19} \mathrm{H}_{27} \mathrm{NO}_{4} \mathrm{~S}, \mathrm{~F} . \mathrm{W} .=365.48$, monoclinic, space group C2/c, $a=17.4458(17) \AA, b=14.1729(13) \AA, c=16.0903(15) \AA, \mathrm{V}=3906.8(6) \AA^{3}, \mathrm{Z}=8$, $\mu(\operatorname{Mok} \alpha)=1.243 \mathrm{~g} / \mathrm{cm}^{3}, \mathrm{~F}(000)=1568, \mathrm{~T}=293 \mathrm{~K}$. The intensity data were collected on a Rigaku AFC7R diffractometer with Mok $\alpha$ radiation $(\lambda=0.71073 \AA$, graphite monochromator) and $12 \mathrm{~kW}$ roataing anode generator. $2 \theta$ range $(3.72-56.56) ; 11587$ measured reflections. The 4583 reflections $[\mathrm{R}(\mathrm{int})=0.0487]$ were used in refinement; good of fit indicator, $0.740 ; \mathrm{R}\left(\mathrm{R}_{\mathrm{w}}\right)=0.0451(0.0776) ; p_{\max }, p_{\min }=0.173,-0.134 \mathrm{e} / \AA^{3}$.

\section{Ethyl $N, N^{\prime}$-di-(p-toluenesulfonyl)piperazine-2-carboxylate (5m)}

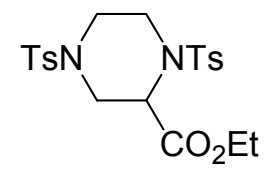

Mp 146-147 ${ }^{\circ} \mathrm{C} .\left(150.9-154.9^{\circ} \mathrm{C} \mathrm{lit.}^{12}\right){ }^{1} \mathrm{H}$ NMR $\left(300 \mathrm{MHz}, \mathrm{CDCl}_{3}\right) \delta 7.64(\mathrm{~d}, J=8.4$ $\mathrm{Hz}), 7.61(\mathrm{~d}, J=8.4 \mathrm{~Hz}, 2 \mathrm{H}), 7.36(\mathrm{~d}, J=8.4 \mathrm{~Hz}, 2 \mathrm{H}), 7.36(\mathrm{~d}, J=8.4 \mathrm{~Hz}, 2 \mathrm{H}) 7.28(\mathrm{~d}$, $J=8.4 \mathrm{~Hz}, 2 \mathrm{H}), 4.74(\mathrm{~m}, 1 \mathrm{H}), 4.22(\mathrm{dt}, J=11.71 .8 \mathrm{~Hz}, 1 \mathrm{H}), 4.09(\mathrm{q}, J=7.2 \mathrm{~Hz}), 3.71$ (m, 2H), $3.45(\mathrm{dt}, J=12.3,3.3 \mathrm{~Hz}, 1 \mathrm{H}), 2.54(\mathrm{dd}, J=3.6,11.7 \mathrm{~Hz}, 1 \mathrm{H}), 2.46(\mathrm{~s}, 3 \mathrm{H})$, $2.42(\mathrm{~s}, 3 \mathrm{H}), 2.36(\mathrm{dd}, J=3.6,11.7 \mathrm{~Hz}, 1 \mathrm{H}), 1.20(\mathrm{t}, 3 \mathrm{H}, J=7.2 \mathrm{~Hz}) .{ }^{13} \mathrm{C}$ NMR $(75$ $\left.\mathrm{MHz}, \mathrm{CDCl}_{3}\right): \delta 167.53,143.97,143.44,136.00,131.88,129.62,129.29,127.36$, $126.95,61.62,54.65,48.10,45.31,41.40,21.24,13.70$. 


\section{$N, N$-Di-(p-toluenesulfonyl)-2-butyrylpiperazine (5n)}

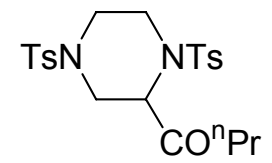

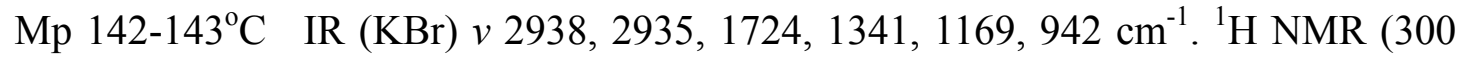
$\left.\mathrm{MHz}, \mathrm{CDCl}_{3}\right) \delta 7.60-7.63(\mathrm{~m}, 4 \mathrm{H}), 7.32(\mathrm{~d}, J=8.7 \mathrm{~Hz}, 2 \mathrm{H}), 7.26(\mathrm{~d}, J=8.7 \mathrm{~Hz}, 2 \mathrm{H})$, $4.60(\mathrm{~m}, 1 \mathrm{H}), 4.22(\mathrm{dt}, J=12.6,1.5 \mathrm{~Hz}, 1 \mathrm{H}), 3.71(\mathrm{dt}, J=13.2,1.5 \mathrm{~Hz}, 1 \mathrm{H}), 3.56(\mathrm{dt}, J$ $=11.5,1.5 \mathrm{~Hz}, 1 \mathrm{H}), 3.33(\mathrm{dd}, J=3.3,12.6 \mathrm{~Hz}, 1 \mathrm{H}), 2.46(\mathrm{~s}, 3 \mathrm{H}), 2.41(\mathrm{~s}, 3 \mathrm{H})$, 2.37-2.59 (m, 3H), $2.31(\mathrm{dt}, J=3.3,11.5 \mathrm{~Hz}, 1 \mathrm{H}), 1.59(\mathrm{~m}, 2 \mathrm{H}), 0.89(\mathrm{t}, J=7.5 \mathrm{~Hz}, 3 \mathrm{H})$.

${ }^{13} \mathrm{C} \mathrm{NMR}\left(75 \mathrm{MHz}, \mathrm{CDCl}_{3}\right): \delta 204.21,144.32,143.68,136.40,131.82,129.85,129.54$, $127.64,127.15,61.15,46.87,45.37,41.77,40.81,21.48,16.68,13.48 . \mathrm{MS}(\mathrm{m} / \mathrm{z}) 394$ $\left(\mathrm{M}^{+}-\mathrm{C}_{3} \mathrm{H}_{6} \mathrm{CO}\right), 393,238(100), 155,91,83,55$. Anal. Calcd for $\mathrm{C}_{22} \mathrm{H}_{28} \mathrm{~N}_{2} \mathrm{O}_{5} \mathrm{~S}_{2}: \mathrm{C}$, 56.87; H, 6.07; N, 6.03. Found C, 56.76; H, 6.15; N, 5.91.

\section{Ethyl $N, N^{\prime}-$ di-( $p$-toluenesulfonyl)diazepane-2-carboxylate (5o)}<smiles>CCOC(=O)C1CN([As])CCC[NH+]1[Sn]</smiles>

Oil. IR (neat) $v 1716,1342,1160,736 \mathrm{~cm}^{-1} .{ }^{1} \mathrm{H}$ NMR $\left(300 \mathrm{MHz}, \mathrm{CDCl}_{3}\right) \delta 7.71(\mathrm{~d}, J=$ $8.1 \mathrm{~Hz}, 2 \mathrm{H}), 7.67(\mathrm{~d}, J=8.1 \mathrm{~Hz}, 2 \mathrm{H}), 7.30(\mathrm{t}, J=7.5 \mathrm{~Hz}, 4 \mathrm{H}), 4.88(\mathrm{t}, J=6.0 \mathrm{~Hz}, 1 \mathrm{H})$, $4.10(\mathrm{~m}, 2 \mathrm{H}), 3.84(\mathrm{dt}, J=15.3,4.2 \mathrm{~Hz}, 1 \mathrm{H}), 3.72(\mathrm{dd}, J=6.6,14.7 \mathrm{~Hz}, 1 \mathrm{H}), 3.60(\mathrm{dd}, J$ $=5.1,14.7 \mathrm{~Hz}, 1 \mathrm{H}), 3.42(\mathrm{ddd}, J=3.6,7.2,15.3 \mathrm{~Hz}, 1 \mathrm{H}), 3.24(\mathrm{~m}, 2 \mathrm{H}), 2.43(\mathrm{~s}, 3 \mathrm{H})$, $2.42(\mathrm{~s}, 3 \mathrm{H}), 1.81(\mathrm{~m}, 2 \mathrm{H}), 1.21(\mathrm{t}, J=7.0 \mathrm{~Hz}, 3 \mathrm{H}) .{ }^{13} \mathrm{C}$ NMR $\left(75 \mathrm{MHz}, \mathrm{CDCl}_{3}\right): \delta$ $169.12,143.53,143.34,136.79,135.44,129.70,129.39,127.07,126.82,61.53,59.29$, 51.19, 49.16, 43.58, 29.14, 21.30, 13.74. MS (m/z) $407\left(\mathrm{M}^{+}-\mathrm{CO}_{2} \mathrm{Et}\right), 325$ (100), 252 , 210, 155, 116, 97, 91. Anal. Calcd for $\mathrm{C}_{22} \mathrm{H}_{28} \mathrm{~N}_{2} \mathrm{O}_{6} \mathrm{~S}_{2}$ : C, 54.98; H, 5.87; N, 5.83. 
Found C, 54.62; H, 5.82; N, 5.80.

\section{Synthesis of piperazine-2-carboxylic acid:}

A suspension of $\mathbf{5 m}(290 \mathrm{mg}, 0.62 \mathrm{mmol})$ in $48 \% \mathrm{HBr}(3 \mathrm{~mL})$ was heated under reflux for $48 \mathrm{hrs}$. The reactant disappeared and white solid precipitated during this period. The mixture was then cooled and the solid was collected by filtration. The filtrate was concentrated (ca $1 \mathrm{~mL})$ and white solid precipitated again. The combined solid was recrystallized from acetone-water to give piperazine-2-carboxylic acid dihydrobromide $128 \mathrm{mg}(71 \%) . \mathrm{Mp} 275^{\circ} \mathrm{C}(\mathrm{d})\left(282^{\circ} \mathrm{C}\right.$ lit. $\left.{ }^{13}\right){ }^{1} \mathrm{H} \mathrm{NMR}\left(300 \mathrm{MHz}, \mathrm{D}_{2} \mathrm{O}\right)$ $\delta 4.21(\mathrm{~m}, 1 \mathrm{H}) 3.94(\mathrm{dd}, J=3.9,14.1 \mathrm{~Hz}, 1 \mathrm{H}), 3.72(\mathrm{~m}, 2 \mathrm{H}), 3.34-3.52(\mathrm{~m}, 3 \mathrm{H})$.

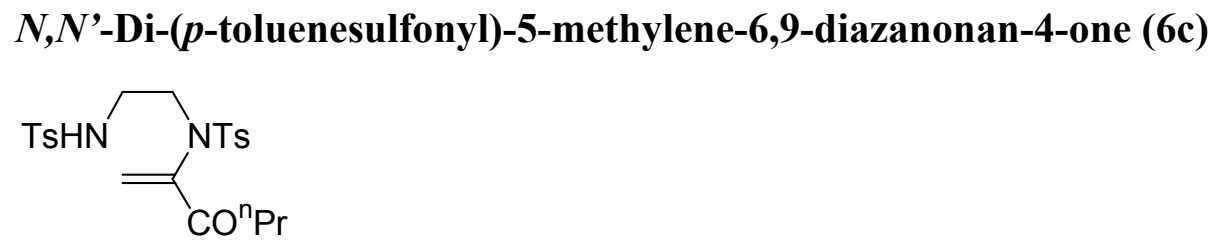

This product ( $\alpha$-adduct) was isolated (22\%) from the reaction of bifunctional nitrogen nucleophile 1d with electron-deficent alkyne $4 \mathbf{f}$ (the reaction condition was similar to that described in Table 1, No. 14, except that the reaction time was shortened to $10 \mathrm{hrs}$ ). Cyclized product $\mathbf{5 n}$ was isolated in $62 \%$ yield in this reaction. The $\alpha$-adduct was used in the control experiment described below.

Oil. IR (neat) $v 3284,2965,1695,1336,1263 \mathrm{~cm}^{-1} .{ }^{1} \mathrm{H}$ NMR $\left(300 \mathrm{MHz}, \mathrm{CDCl}_{3}\right) \delta 7.71$ (d, $J=7.6 \mathrm{~Hz}, 2 \mathrm{H}), 7.60(\mathrm{~d}, J=7.6 \mathrm{~Hz}, 2 \mathrm{H}), 7.29(\mathrm{~d}, J=8.1 \mathrm{~Hz}, 4 \mathrm{H}), 6.10(\mathrm{~d}, J=1.5$ $\mathrm{Hz}, 1 \mathrm{H}), 5.64(\mathrm{t}, J=6.0 \mathrm{~Hz}, 1 \mathrm{H}), 5.53(\mathrm{t}, J=1.5 \mathrm{~Hz}, 1 \mathrm{H}), 3.40(\mathrm{t}, J=6.0 \mathrm{~Hz}, 2 \mathrm{H}), 3.02$ $(\mathrm{dd}, J=6.0,11.1 \mathrm{~Hz}, 2 \mathrm{H}), 2.65(\mathrm{t}, J=7.5 \mathrm{~Hz}, 2 \mathrm{H}), 2.42(\mathrm{~s}, 3 \mathrm{H}), 1.61(\mathrm{~m}, 2 \mathrm{H}), 0.91(\mathrm{t}, J$ $=7.5 \mathrm{~Hz}, 3 \mathrm{H}) .{ }^{13} \mathrm{C} \mathrm{NMR}\left(75 \mathrm{MHz}, \mathrm{CDCl}_{3}\right): \delta 198.99,144.57,144.25,143.28,136.74$, $134.44,129.59,127.80,127.06,124.74,49.55,41.56,40.14,21.52,21.44,17.32$, 
13.56. MS (m/z) $393\left(\mathrm{M}^{+}-\mathrm{COC}_{3} \mathrm{H}_{7}\right), 309,280,155,126,91,65$. Anal. Calcd for $\mathrm{C}_{22} \mathrm{H}_{28} \mathrm{NO}_{5} \mathrm{~S}_{2}: \mathrm{C}, 56.87 ; \mathrm{H}, 6.07 ; \mathrm{N}, 6.03$. Found C, 56.77; H, 6.12; N, 5.91.

\section{Control experiment:}

\section{Reaction of 6a in the presence or in the absence of $\mathrm{Ph}_{3} \mathrm{P}$ :}

A solution of $6 \mathbf{a}(25 \mathrm{mg}, 0.11 \mathrm{mmol})$ and $\mathrm{Ph}_{3} \mathrm{P}(5.8 \mathrm{mg}, 0.022 \mathrm{mmol})$ in toluene was heated at $110^{\circ} \mathrm{C}$ for $48 \mathrm{hrs}$. The solvent was removed under reduced pressure and the residue was purified by flash chromatography on silica gel (ethyl acetate:petroleum ether $=2: 1)$ to give $\mathbf{5 a}(23 \mathrm{mg}), 92 \%$.

Heating a solution of $\mathbf{6 a}$ in toluene at $110^{\circ} \mathrm{C}$ in the absence of $\mathrm{Ph}_{3} \mathrm{P}$ gave disordered results.

\section{Reaction of $6 \mathrm{c}$ in the presence or in the absence of $\mathrm{Ph}_{3} \mathrm{P}$ :}

A solution of $6 \mathbf{c}(28 \mathrm{mg}, 0.06 \mathrm{mmol})$ and $\mathrm{Ph}_{3} \mathrm{P}(3.1 \mathrm{mg}, 0.012 \mathrm{mmol})$ in $0.5 \mathrm{ml}$ toluene- $\mathrm{CH}_{3} \mathrm{CN}$ (v:v=4:1) was heated at $80^{\circ} \mathrm{C}$ for $17 \mathrm{hrs}$. The solvent was removed under reduced pressure and the residue was purified by flash chromatography on silica gel (ethyl acetate:petroleum ether $=2: 1)$ to give $5 \mathrm{n}(23 \mathrm{mg}), 82 \%$.

No reaction occurred in the absence of $\mathrm{Ph}_{3} \mathrm{P}$.

\section{References:}

(1) Searle, G. H.; Geue, R. L.; Aust. J. Chem. 1984, 37, 959.

(2) Buono, G.; Synthesis 1981, 11, 872.

(3) Hashmi, A. S. K.; Ruppert, T. L.; Knöfel, T.; Bats, J. W.; J. Org. Chem. 1997, 62, 7295.

(4) Braude, E. A.; Jones, E. R. H.; Sondheimer, F.; Toogood, J. B.; J. Chem. Soc. 
1949, 607.

(5) Curtin, D. Y.; Crump, J. W.; J. Am. Chem. Soc. 1958, 80, 1922.

(6) Brandsma, L.; Preparative Acetylenic Chemistry; 2nd ed., Elsevier: Amsterdam, 1988, p101.

(7) Mori, A.; Ishihara, K.; Arai, I.; Yamamoto, H; Tetrahedron 1987, 43, 755.

(8) Jung, M. E.; Buszek, K. R.; J. Am. Chem. Soc. 1988, 110, 3965.

(9) Bowden, K.; Jones, E. R. H; J. Chem. Soc. 1946, 52

(10) Saari, W. S.; Raab, A. W.; King, S. W.; J. Org. Chem. 1971, 36, 1711.

Bach, F. L.; Krushner, S.; Williams, J. H. J. Am. Chem. Soc. 1955, 77, 6049. 

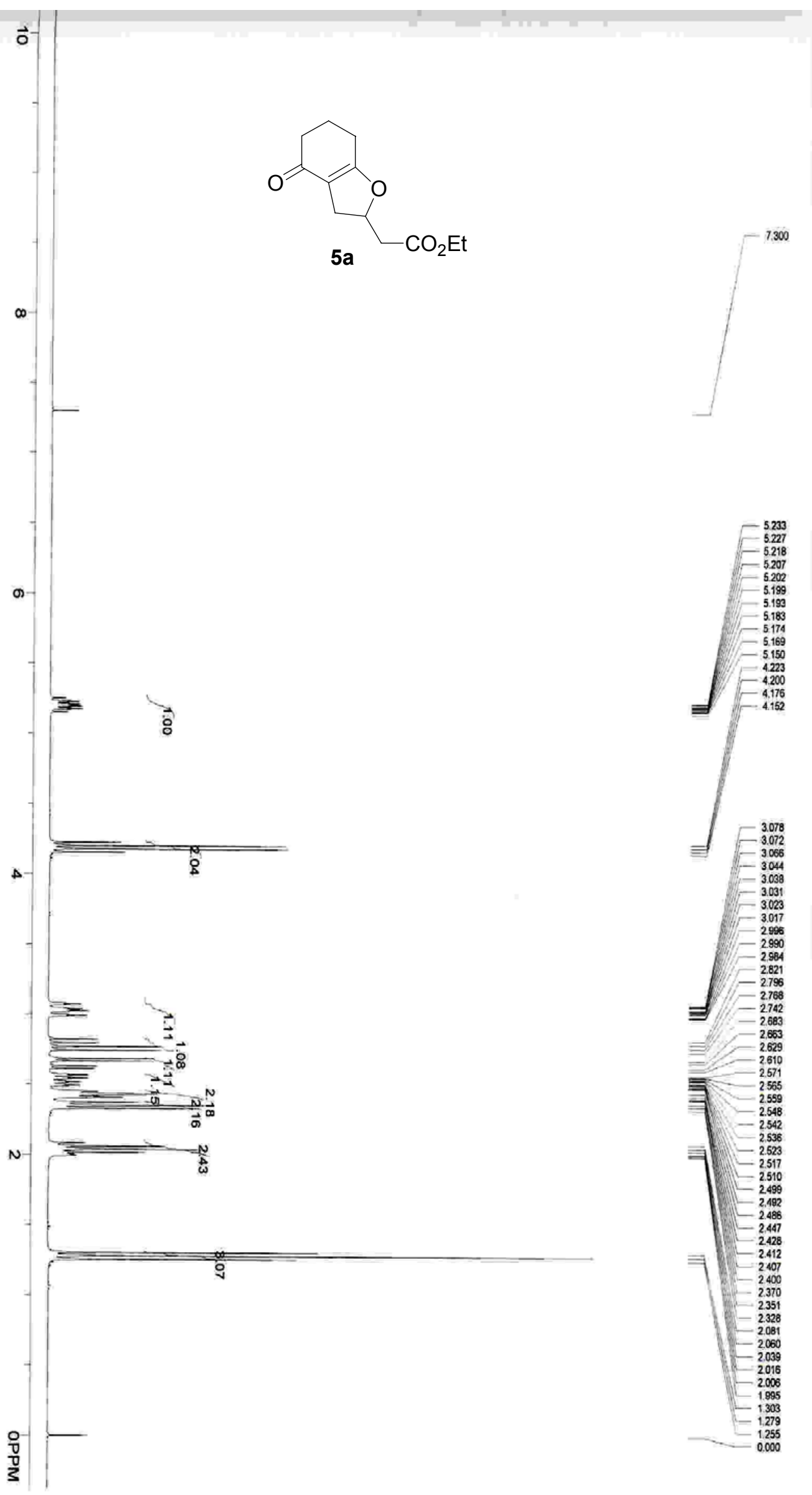

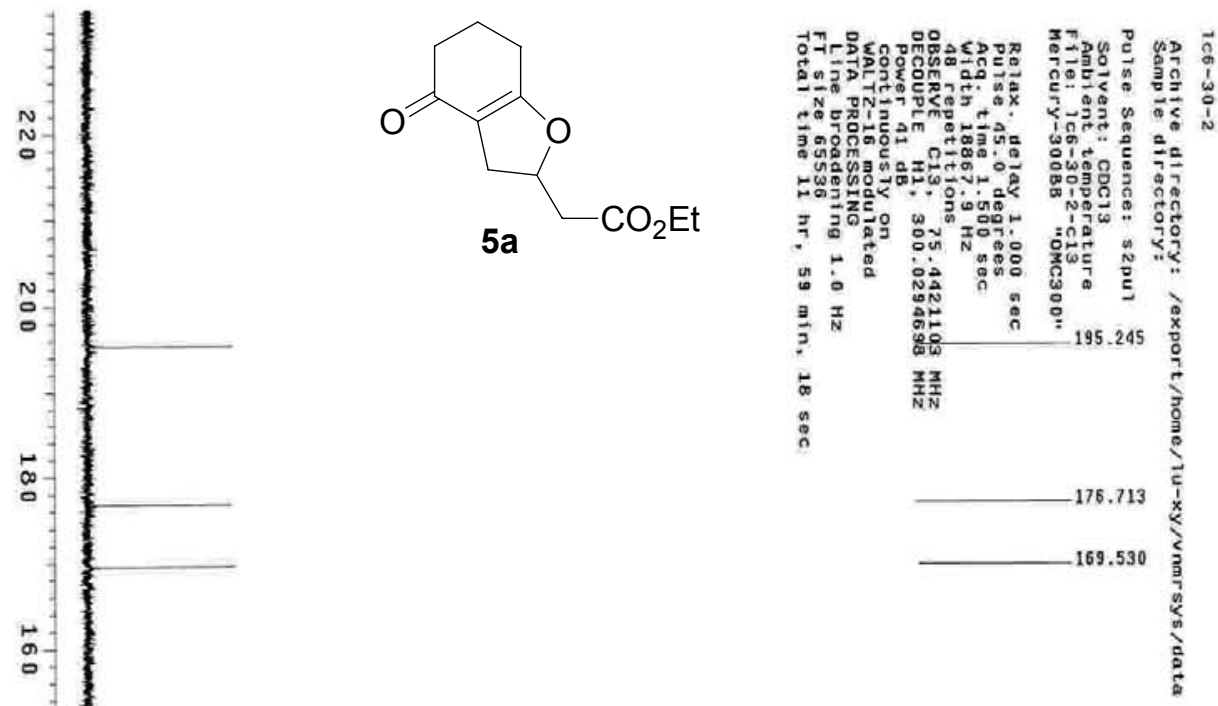

\section{$\mathrm{O}_{2} \mathrm{Et}$}

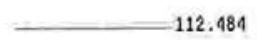

$\cong$
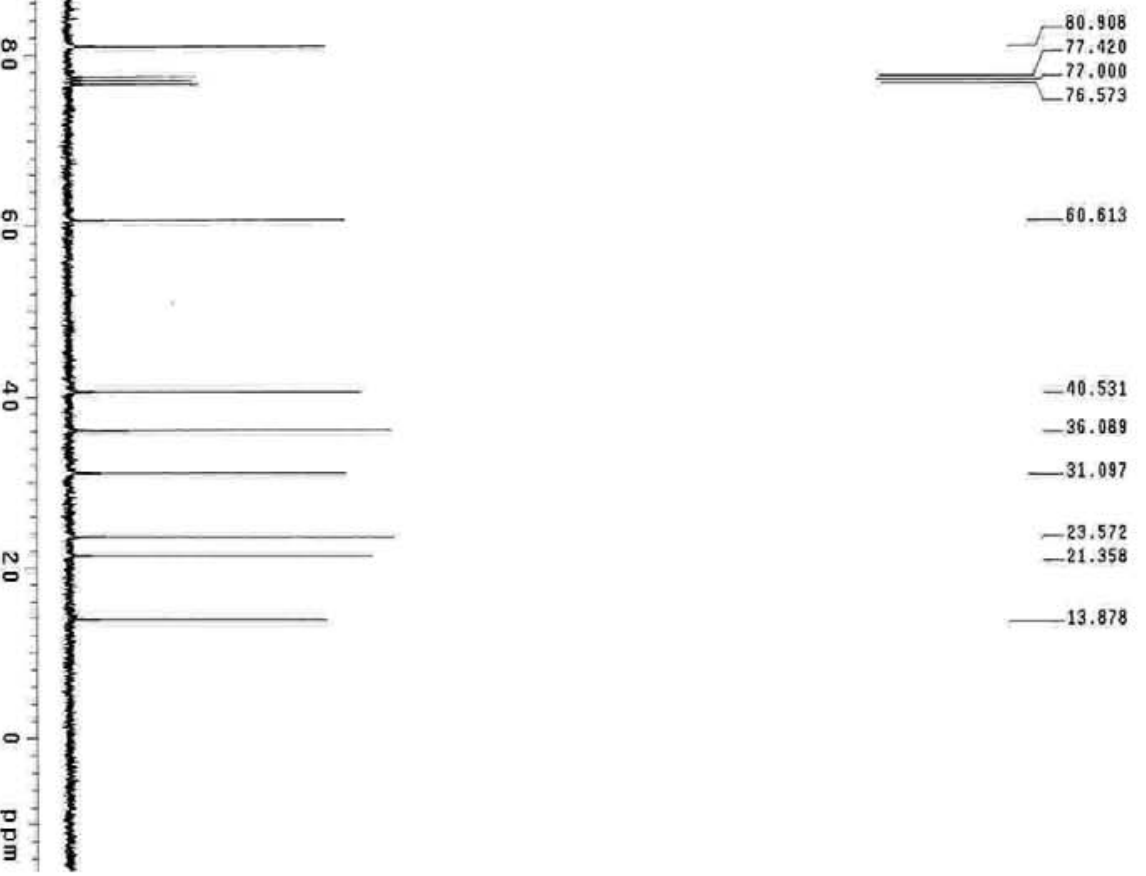

$-40.531$

$-36.089$

- 31.097

-23.572
-21.358

$-13.878$ 


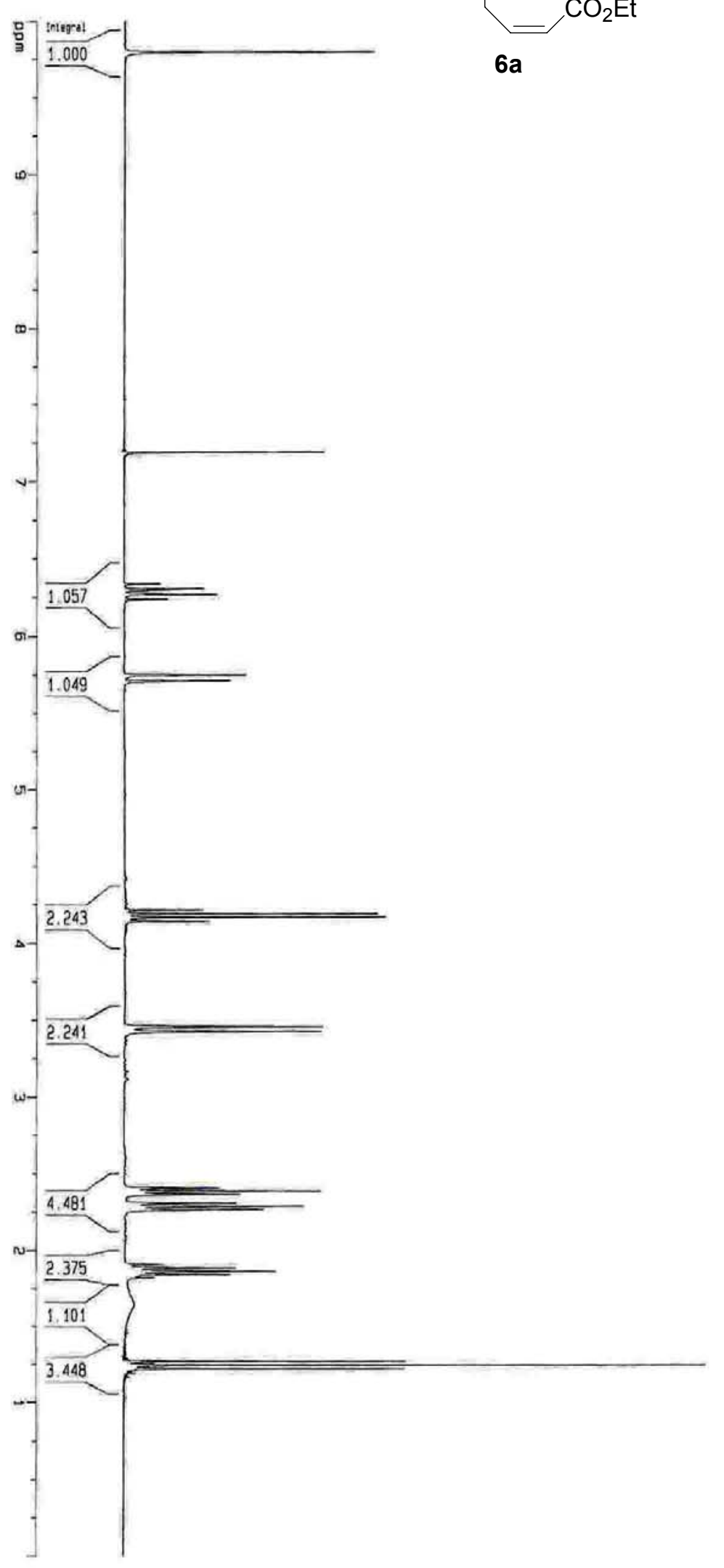

$\mathrm{Hz}^{2}$
-2940.27

$-1892.66$

$-1882.03$

$-1881.38$

$-1726.04$

$-1725.27$

$-1714.75$

$-1713.98$

1265.78

$-1265.15$

$-1258.63$

$-1257.99$

$-1251.48$

$-1250.83$

$-1244.33$

$-1243.69$

$-1038.83$

$-1029.61$

723.00

715.64

- 710.38

- 693.61

687.10

- 680.38

566.18

- 559.55

$-553.17$

381.35

380.69

374.21

373.54

$-367.05$

366.39 

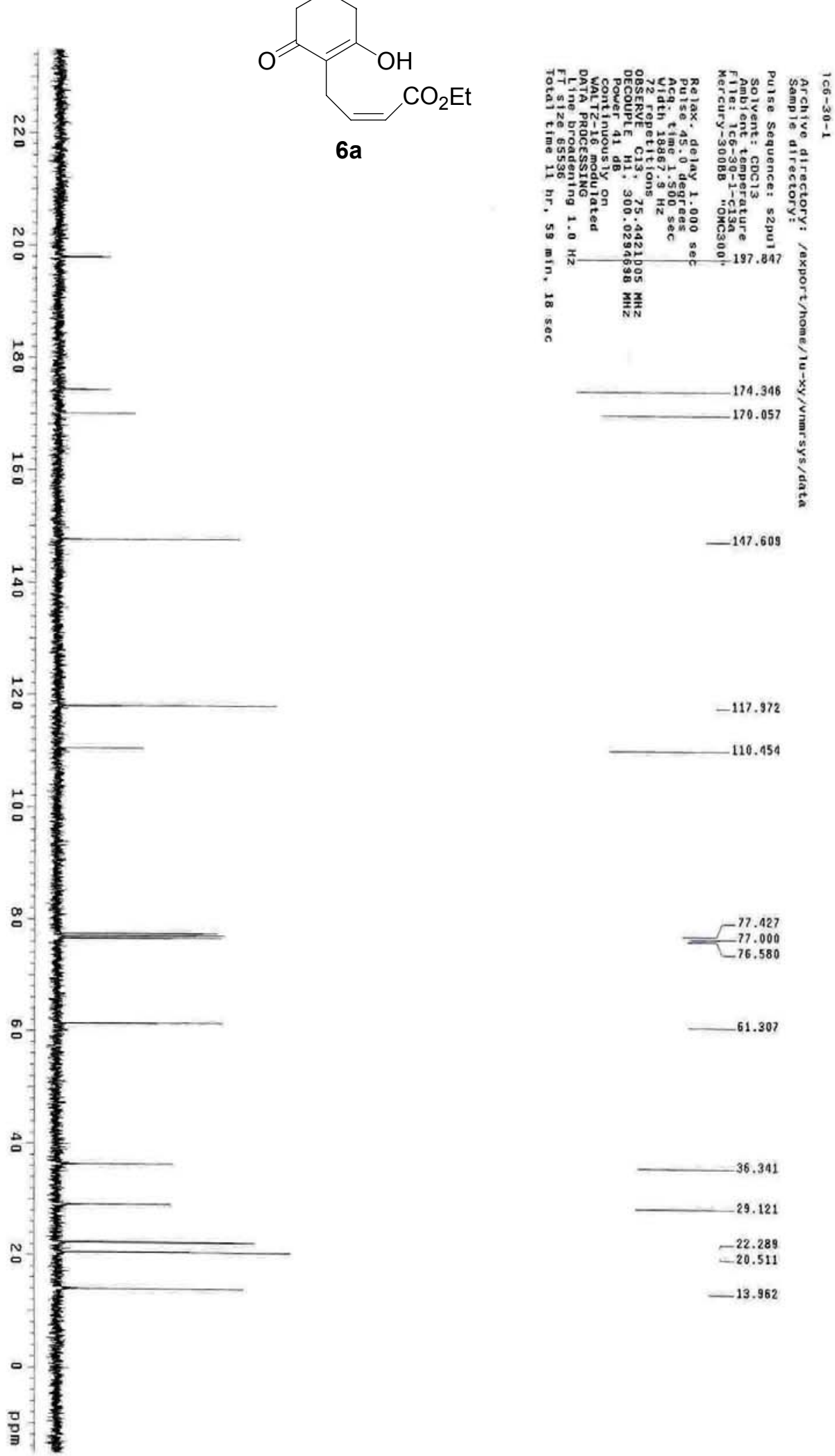


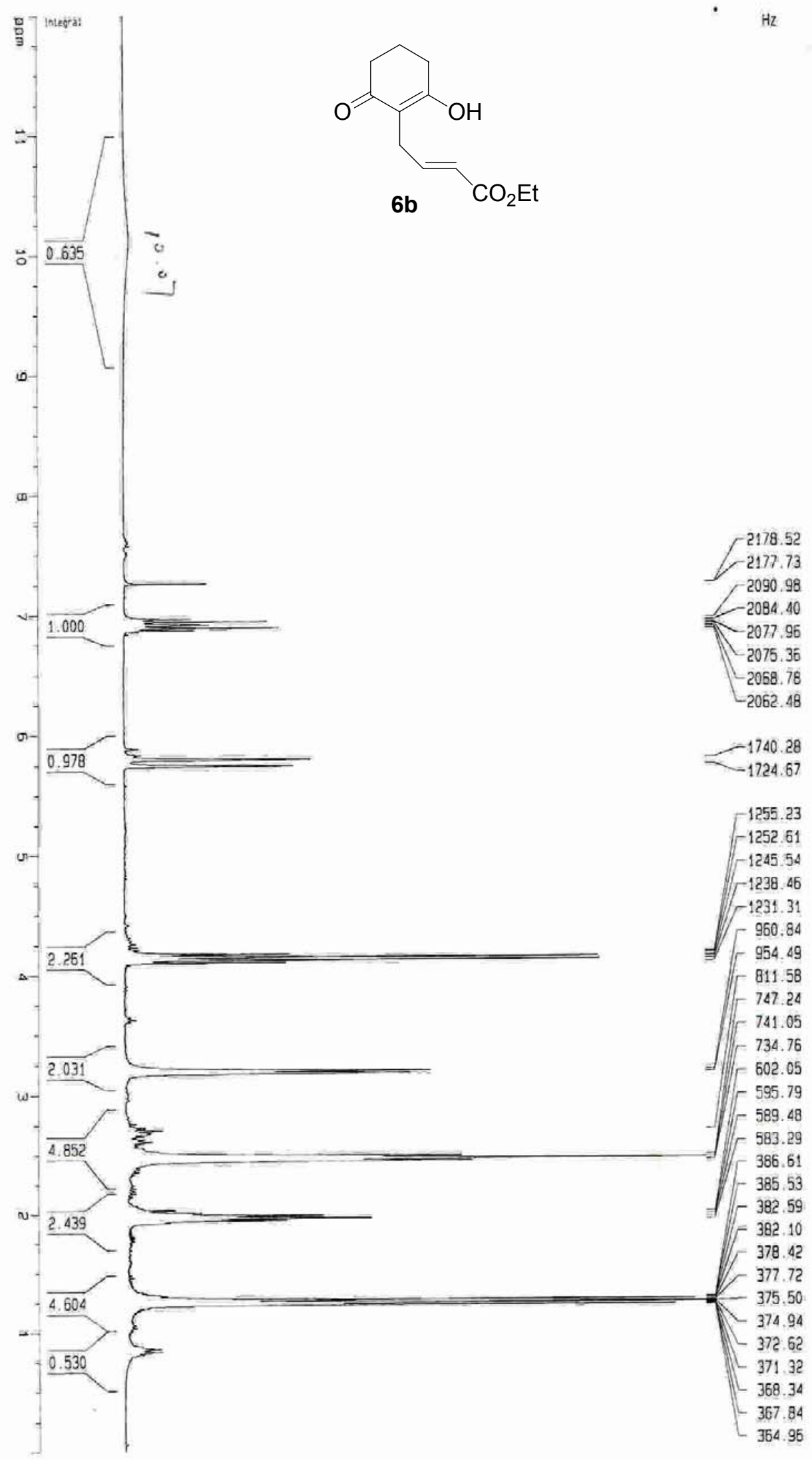



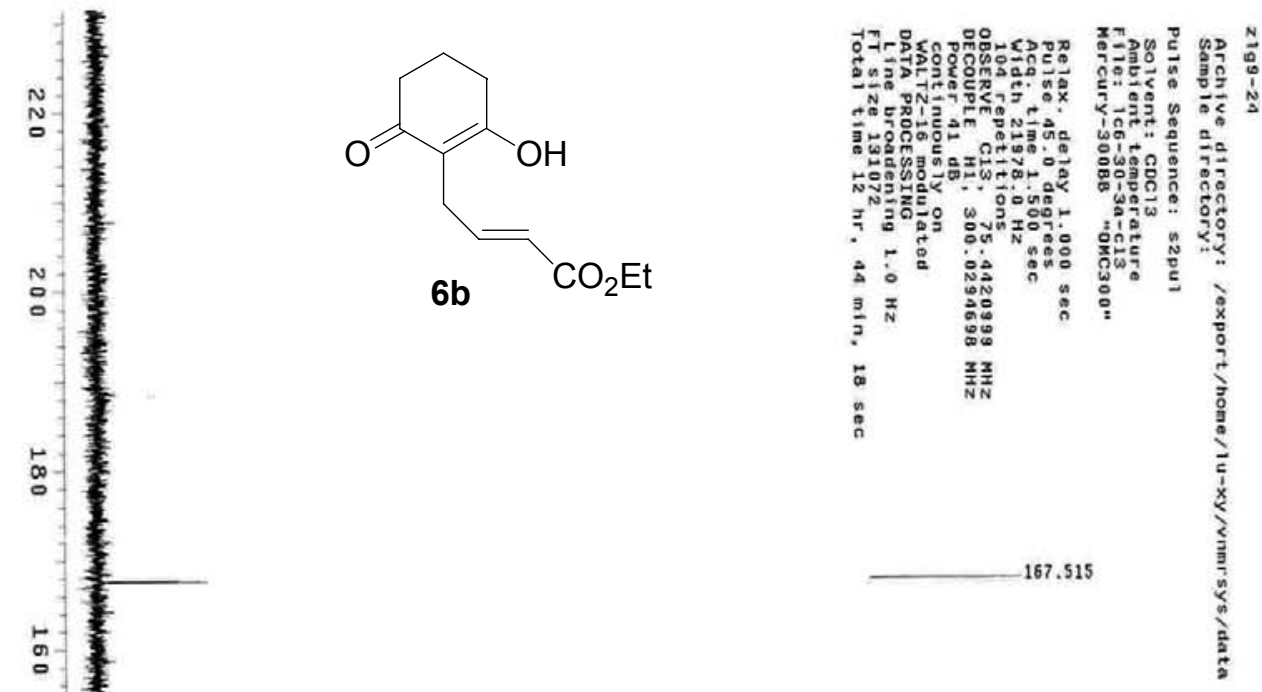

क

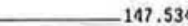

‥

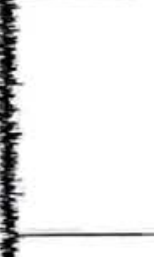

:.

a
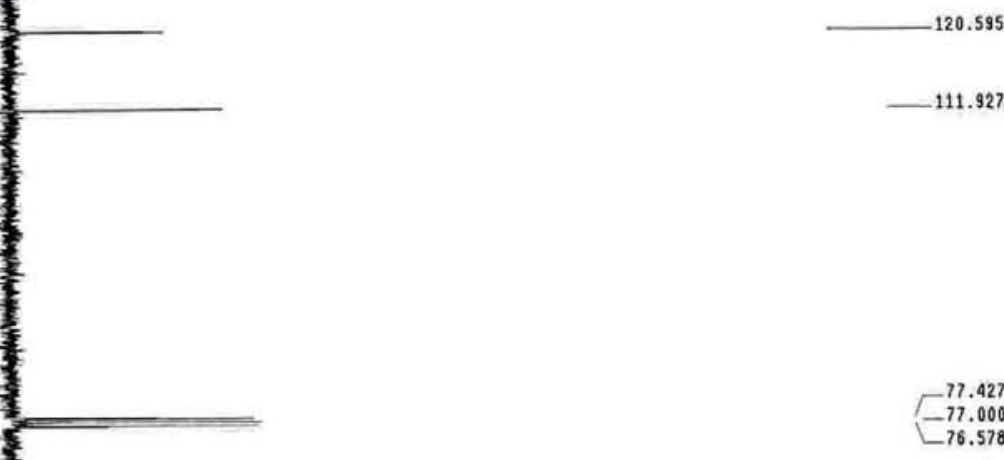

:

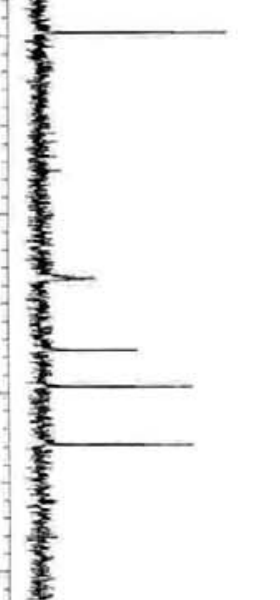

$-111.927$

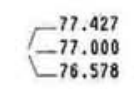

60.299

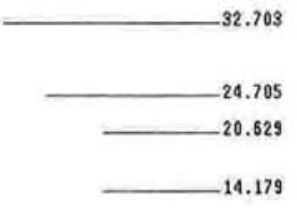




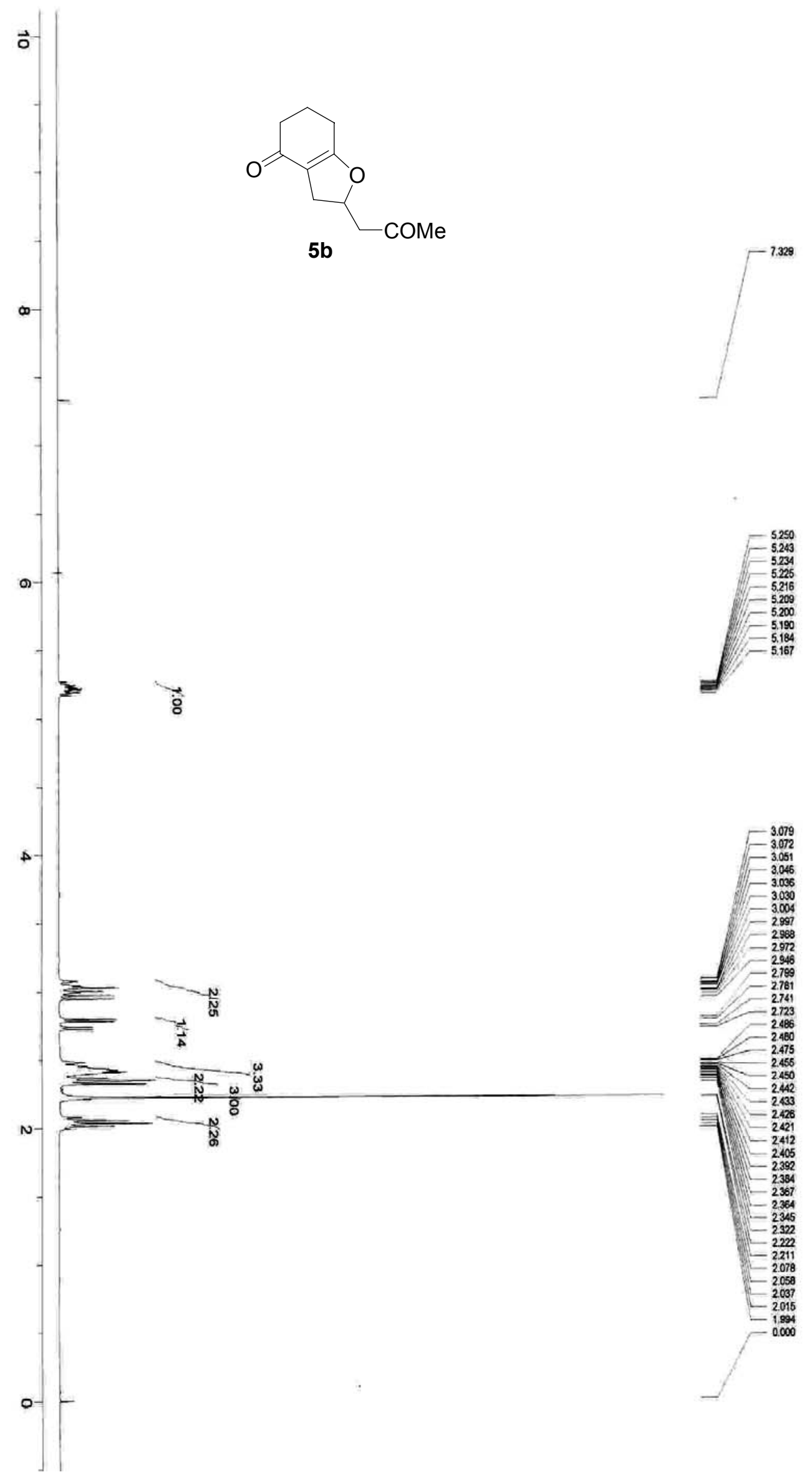




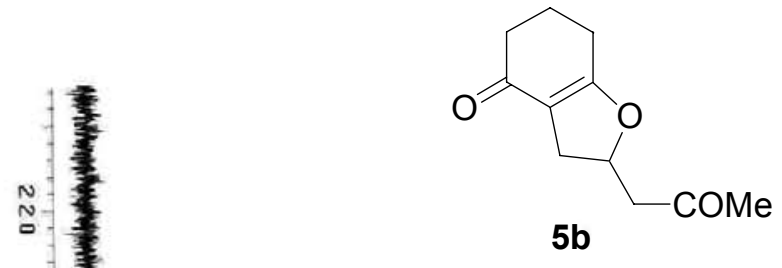

ㄱ.

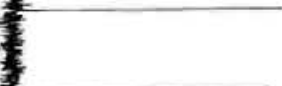

204.999

$\stackrel{b}{\infty}$

妾

。

-

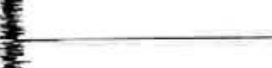

176.835

$\stackrel{N}{N}$

-

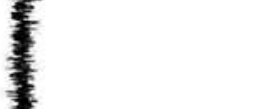

112.606

$\infty$

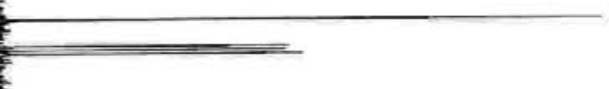

$-77.427$

77.000
-76.580

a

t

类

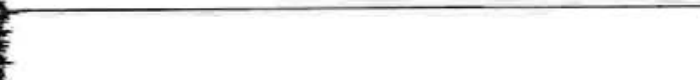

$-36.173$

$-31.426$

$-23.679$

N

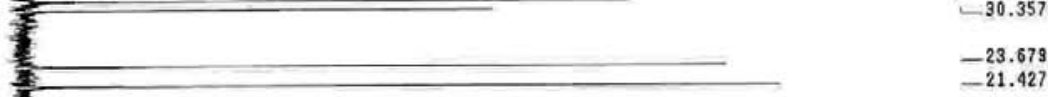

-

뭌

等 


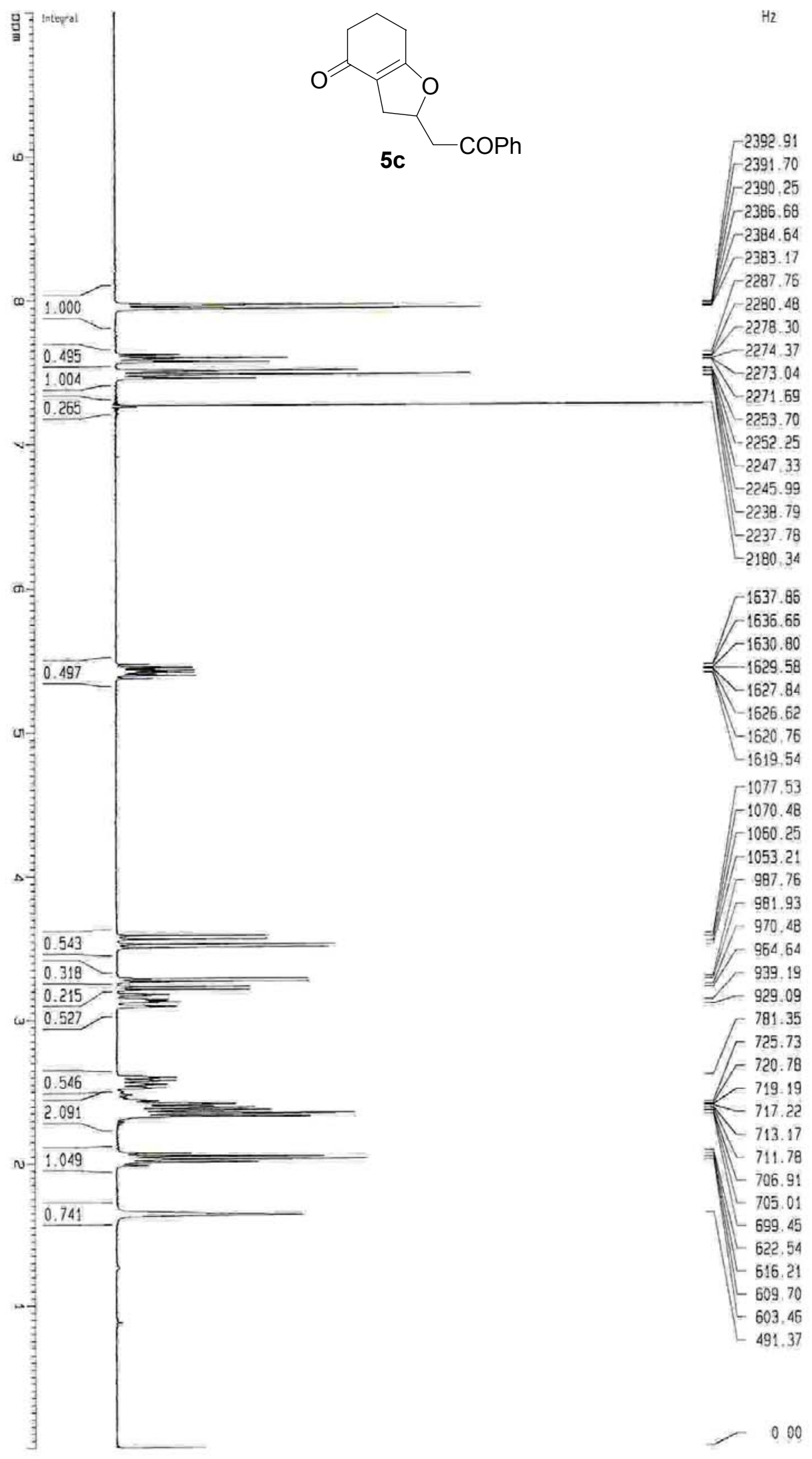



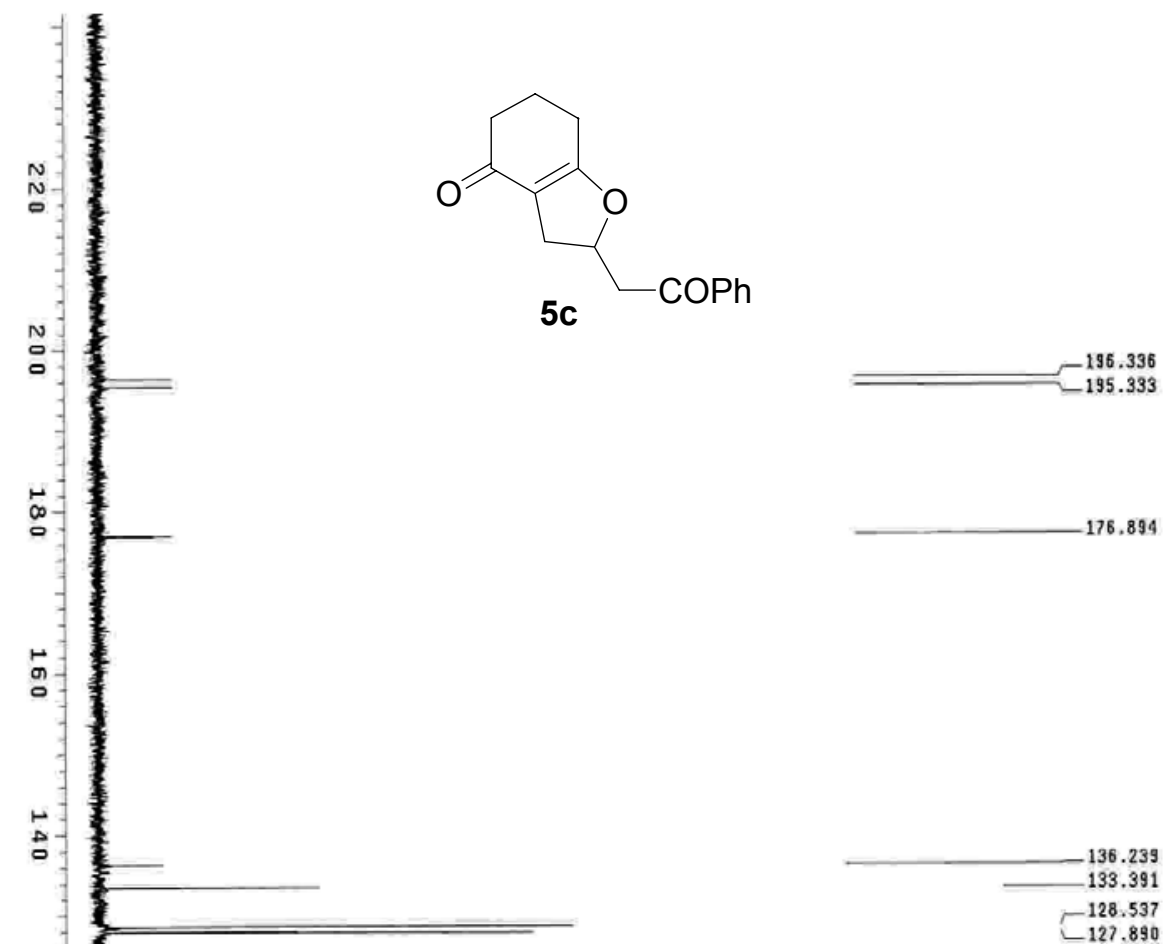

$\stackrel{\leftarrow}{N}$

a.

冓:

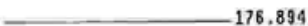

g

然

$\overrightarrow{0}$

$-112.736$

$:$

$\infty$
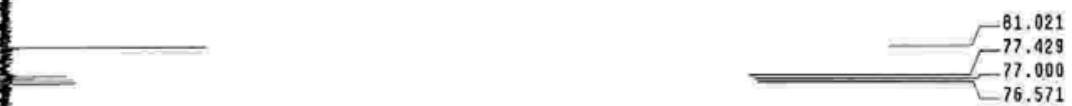

a-

$\circ$

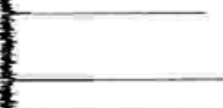

$-44.444$

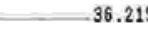

$-31.628$

N.

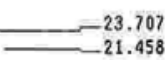

믈 =

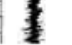



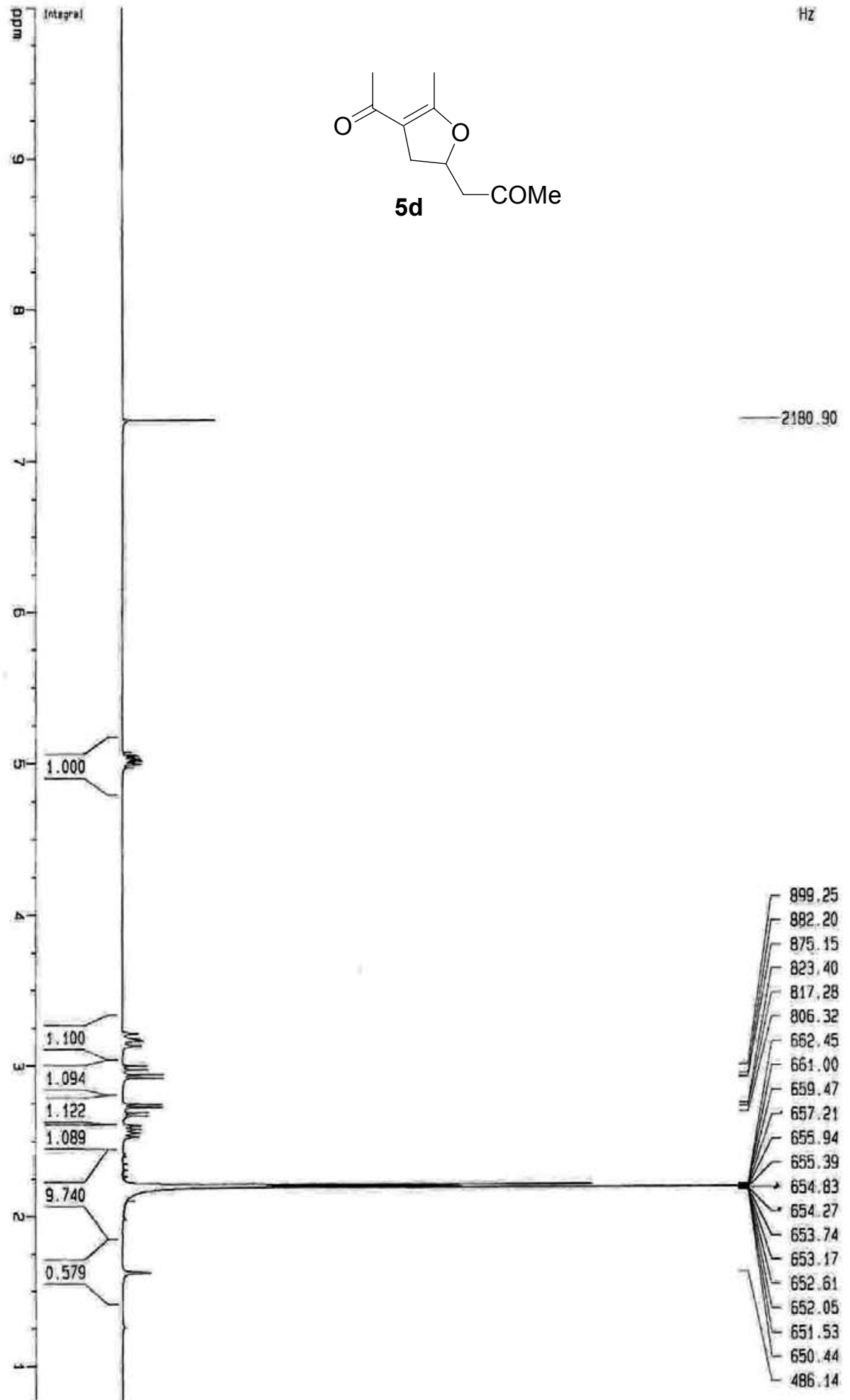

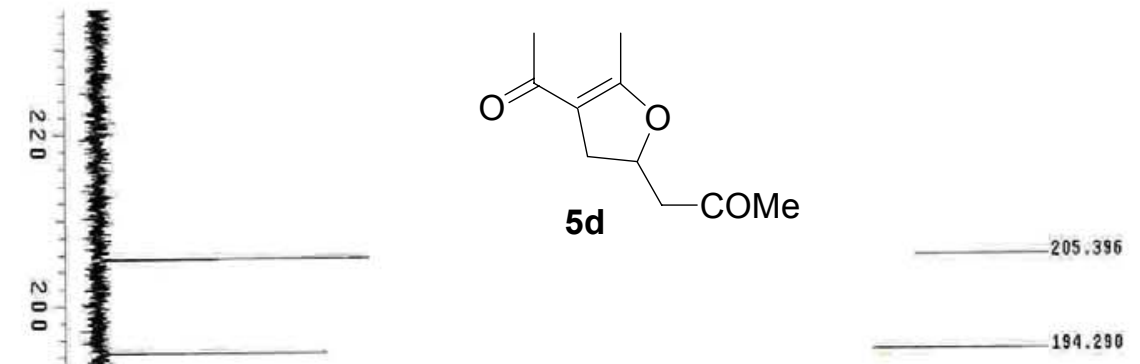

ㅇ.

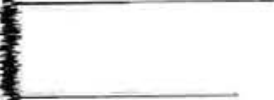

占

$-$

a

N.

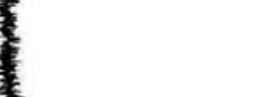

111.614

a

$\stackrel{\infty}{a}$
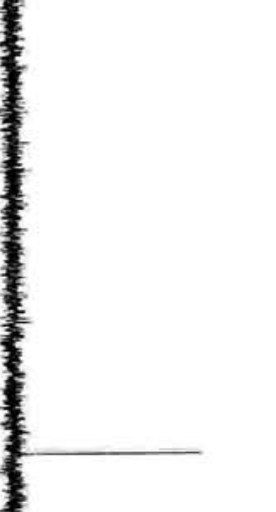

$-166.928$
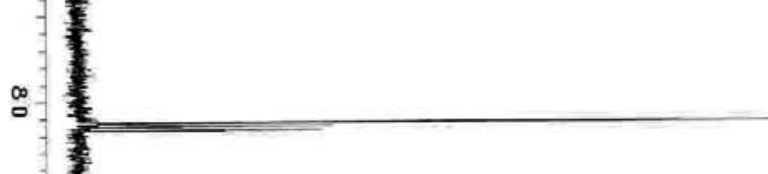

$-77.412$

77.000
-76.580

。

$-$

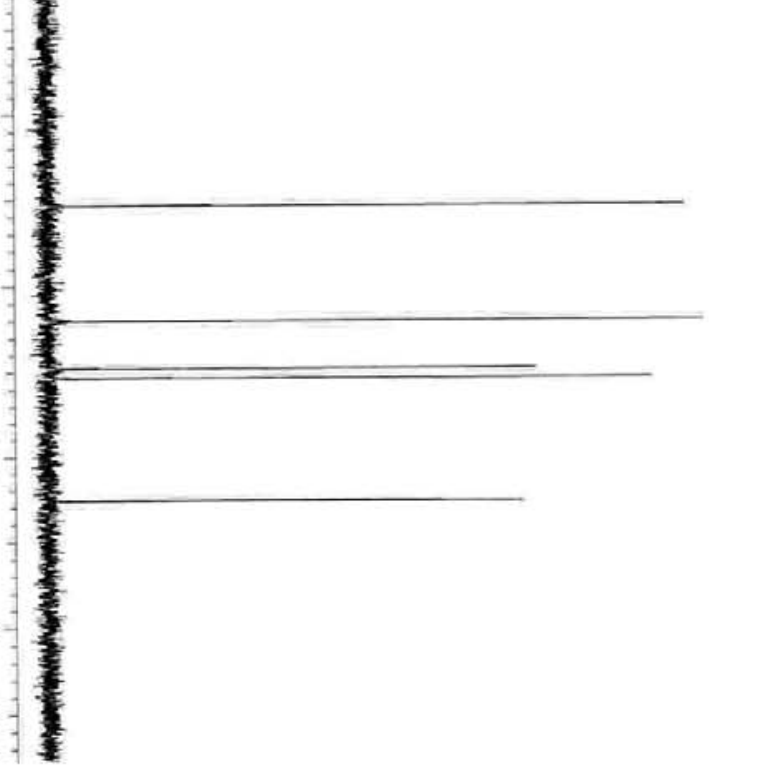

$-49.248$

$-35.952$

$-30.403$

$\subset_{29.220}$

$-14.733$ 


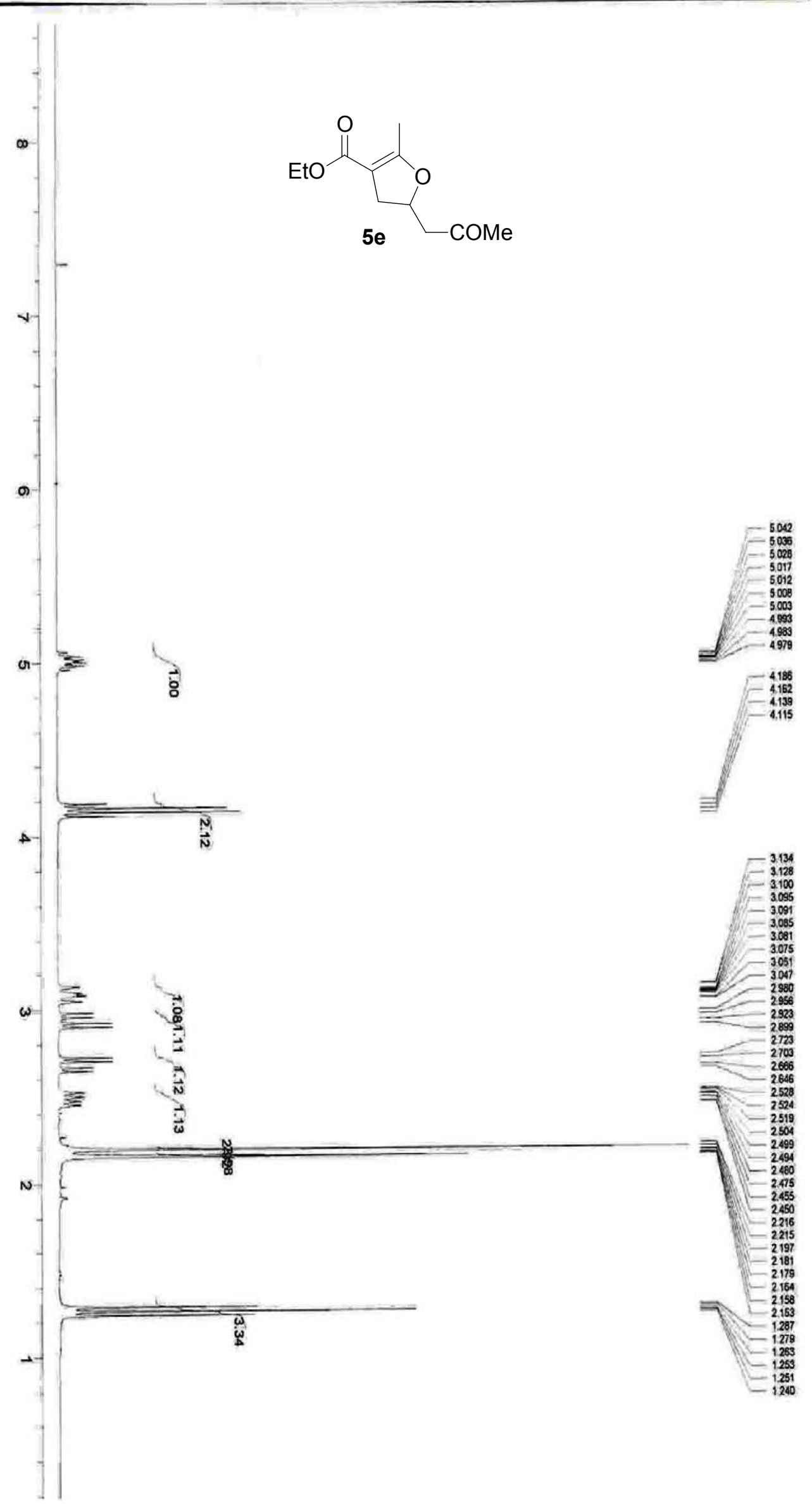



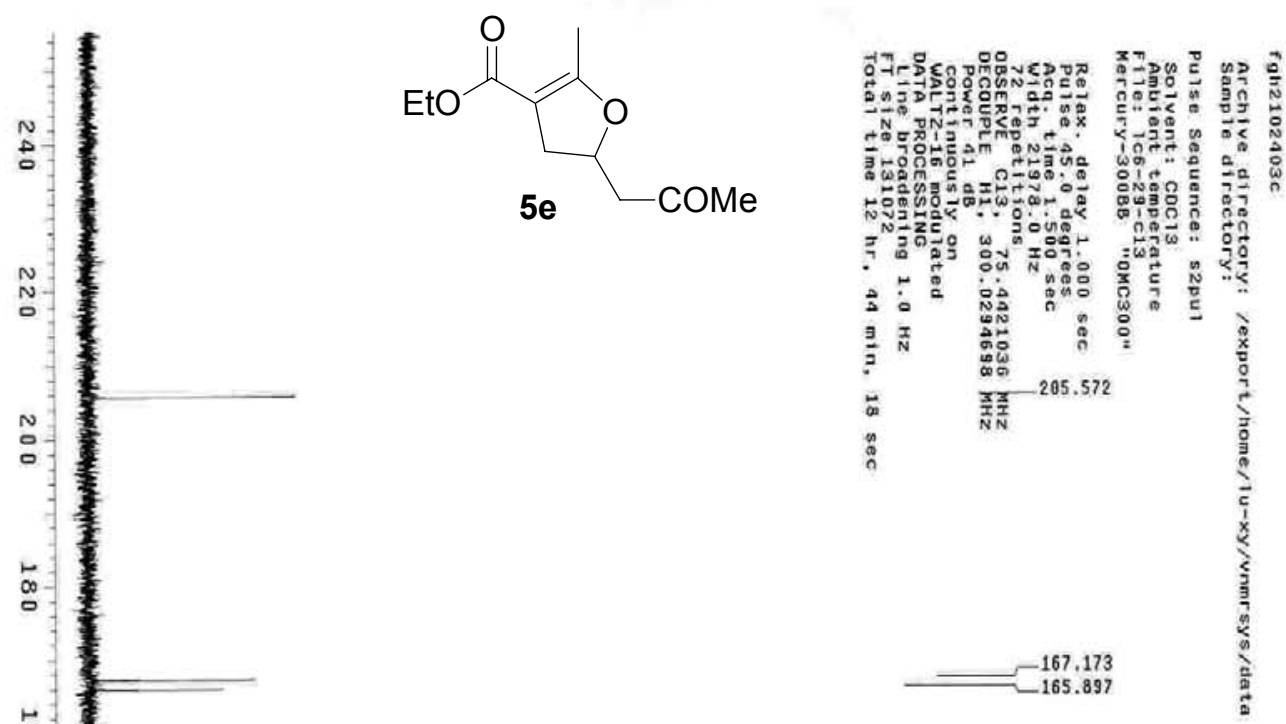

$\therefore-$

:

:

N

:

$-101.516$

o.

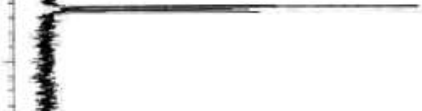

-77.422
$-\quad-77.373$
-77.000

-77.000
-76.573

$-59.321$

$-49.395$

:

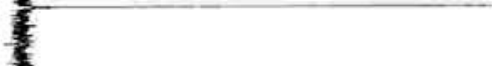

$-35.299$

N-

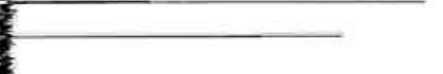

$-30.475$

14.268
-13.890

$\circ$

1

뭅 


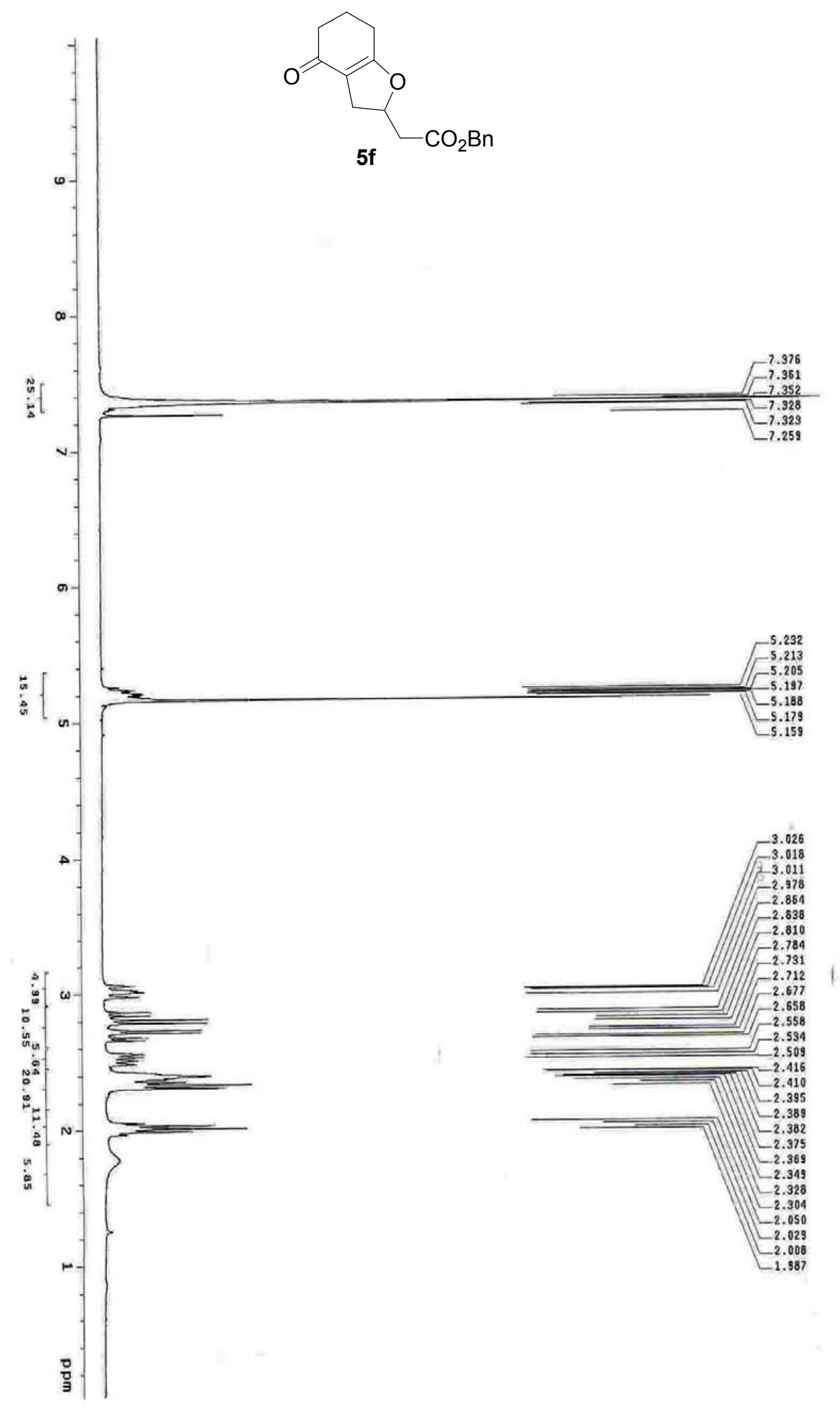




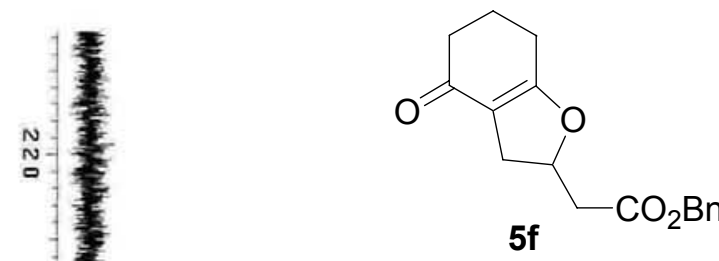

$-195.382$

a-

:

$\stackrel{\circ}{\circ}$

N-
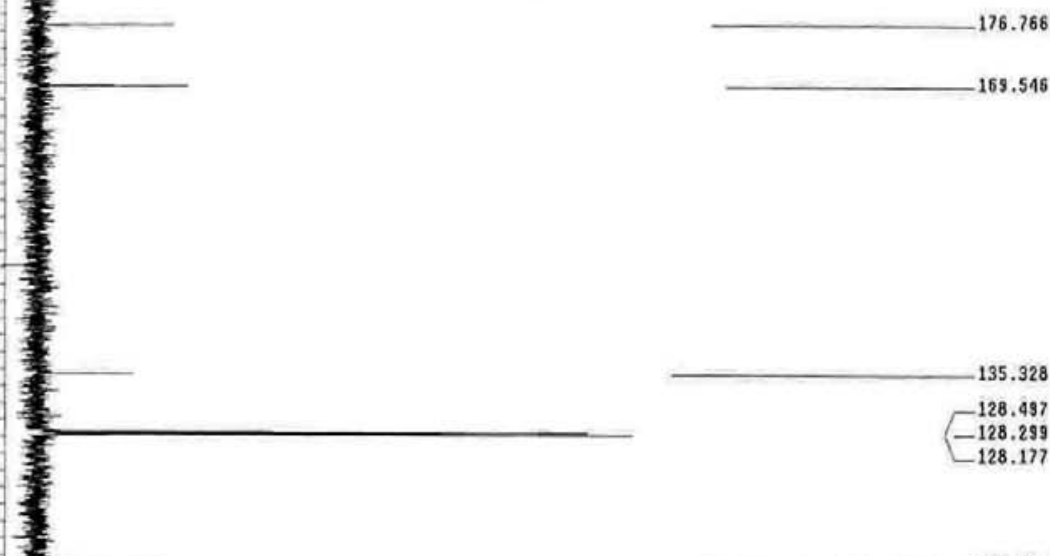

:

$\stackrel{\infty}{\circ}$
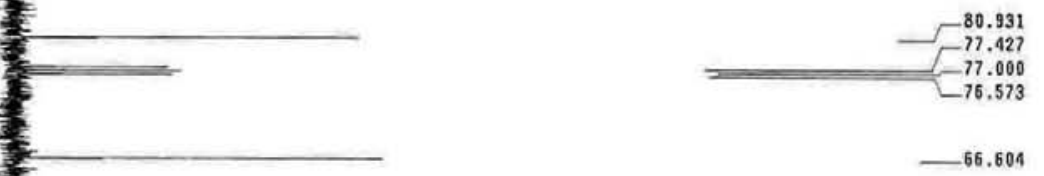

ㅇ
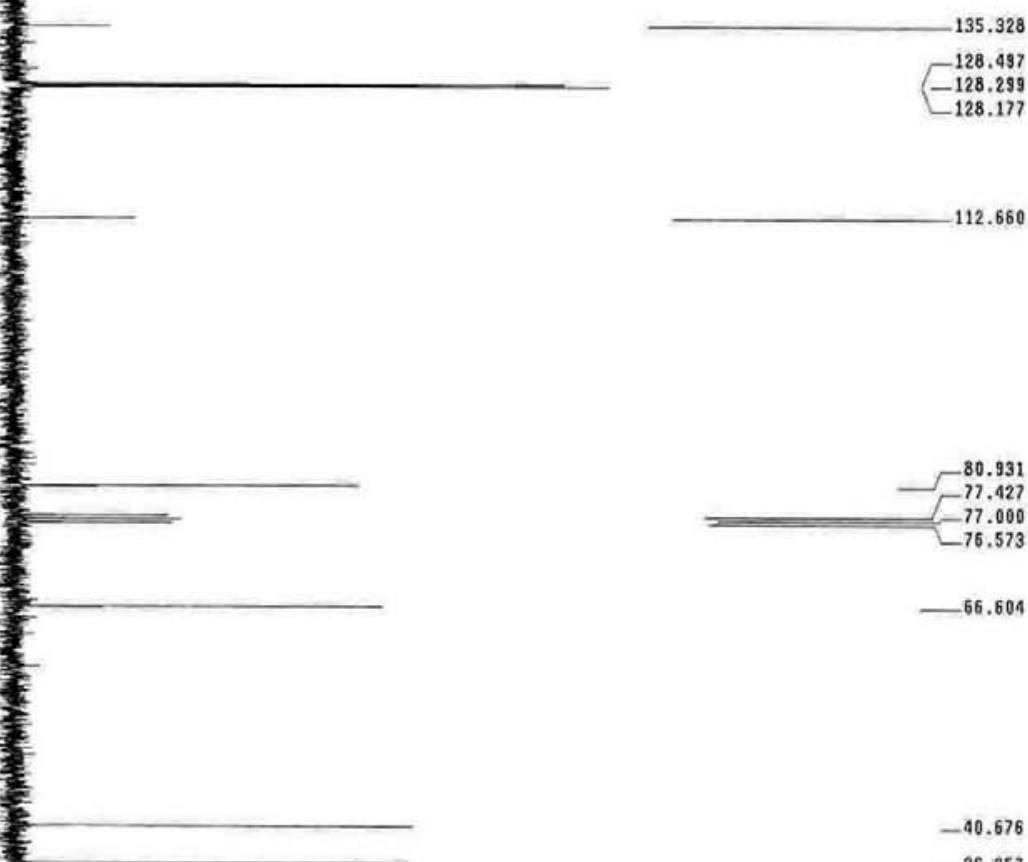

$-40.676$

$-36.257$

$-31.296$

-23.709
-21.488

.

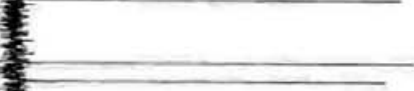

0

g

‥ 转 

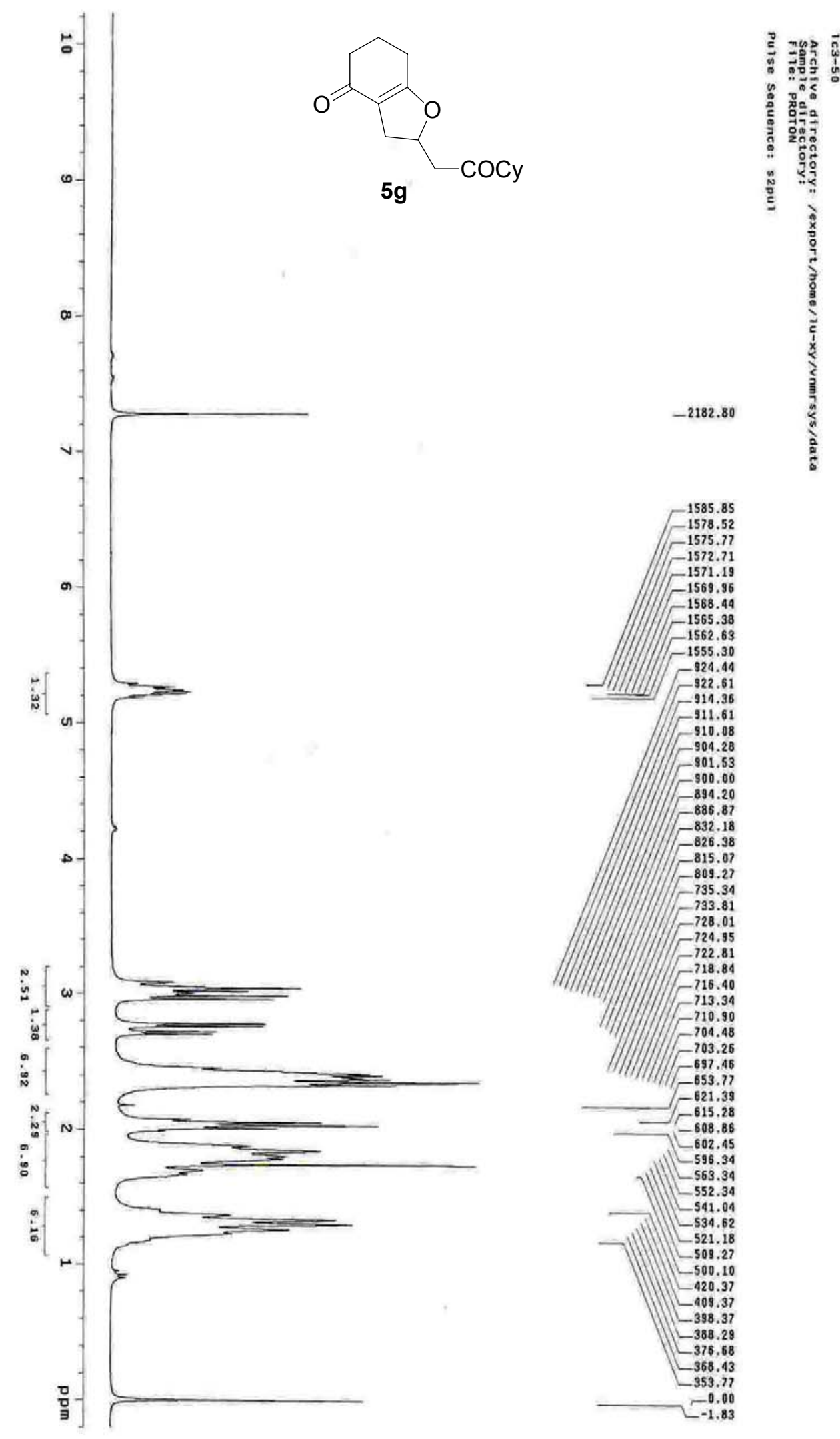


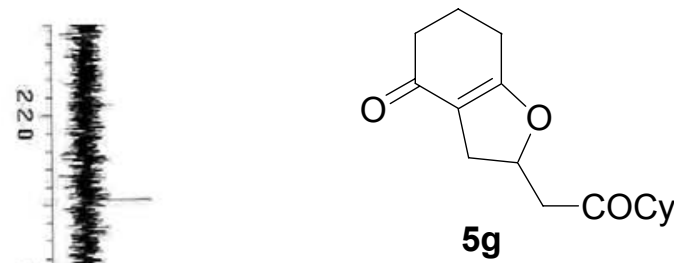

$-210.511$

195.810

112.841

음

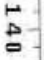

‥

o
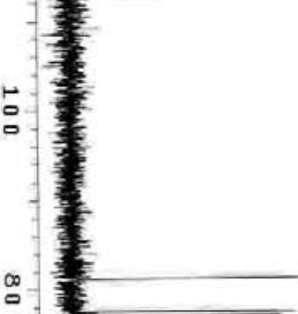

81.150
77.421

$C_{76.579}^{77.000}$
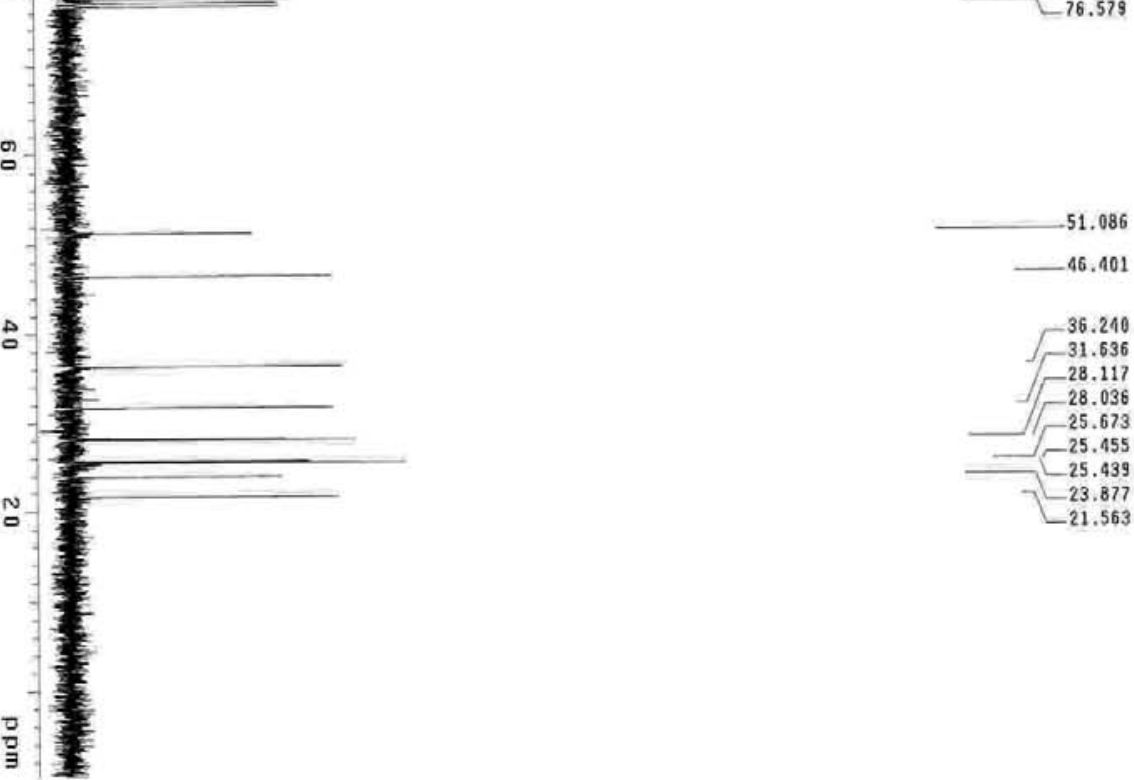

$-21.563$ 

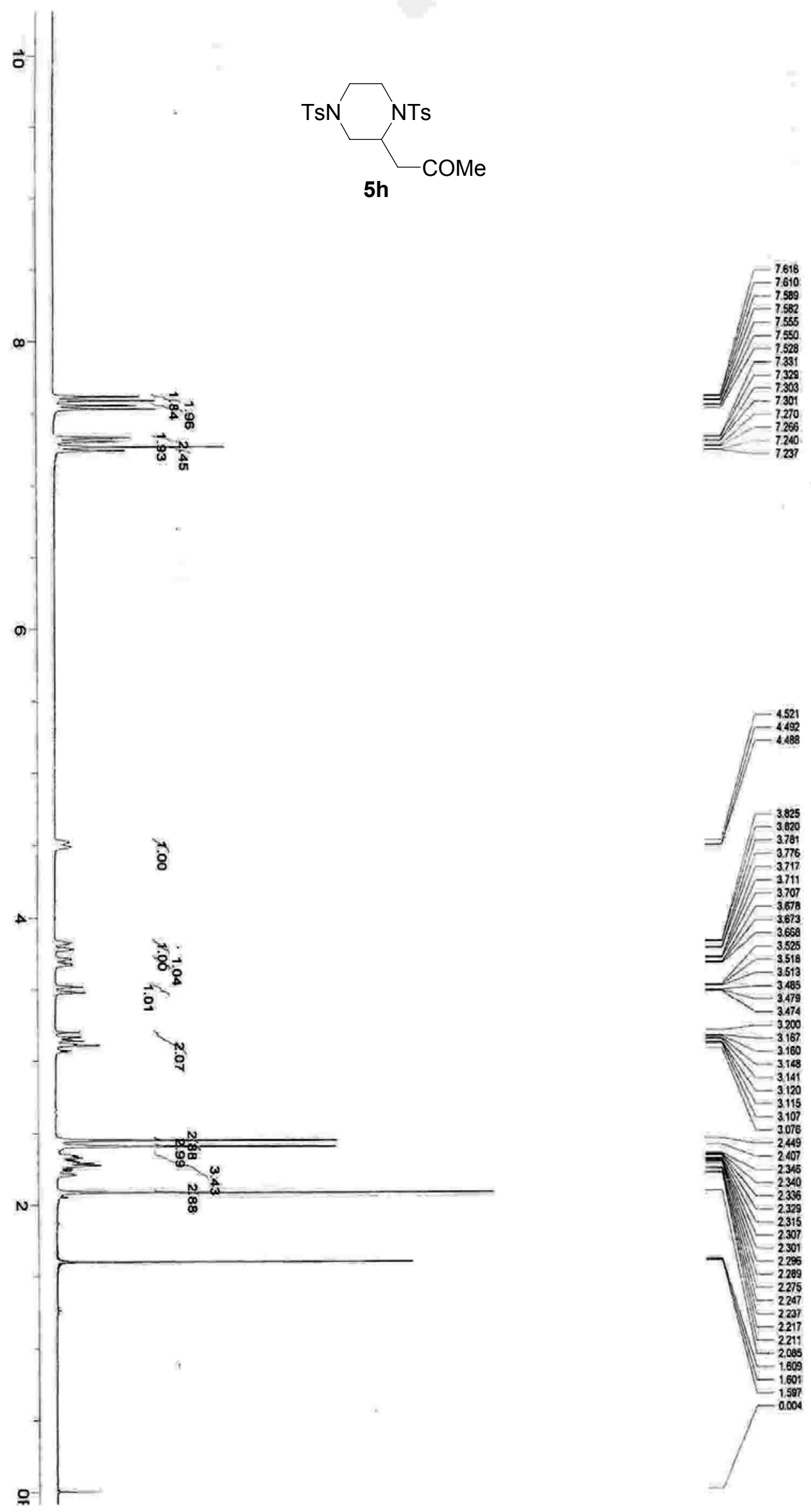
3.825
3.220
3781
3.776
3717
3711
3707
3.678
3873
3.650
3.525
3518
3.513
3485
3.479
3474
3200 $\begin{array}{r}-3200 \\ -3167 \\ \hline-3160\end{array}$ 3.160
-3.148
-3.148 $\begin{array}{r}\square \\ -3.120 \\ -3115 \\ -3.107 \\ -3.076 \\ -2449 \\ \hline\end{array}$

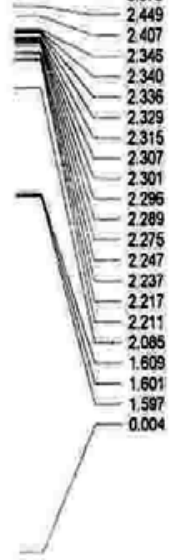



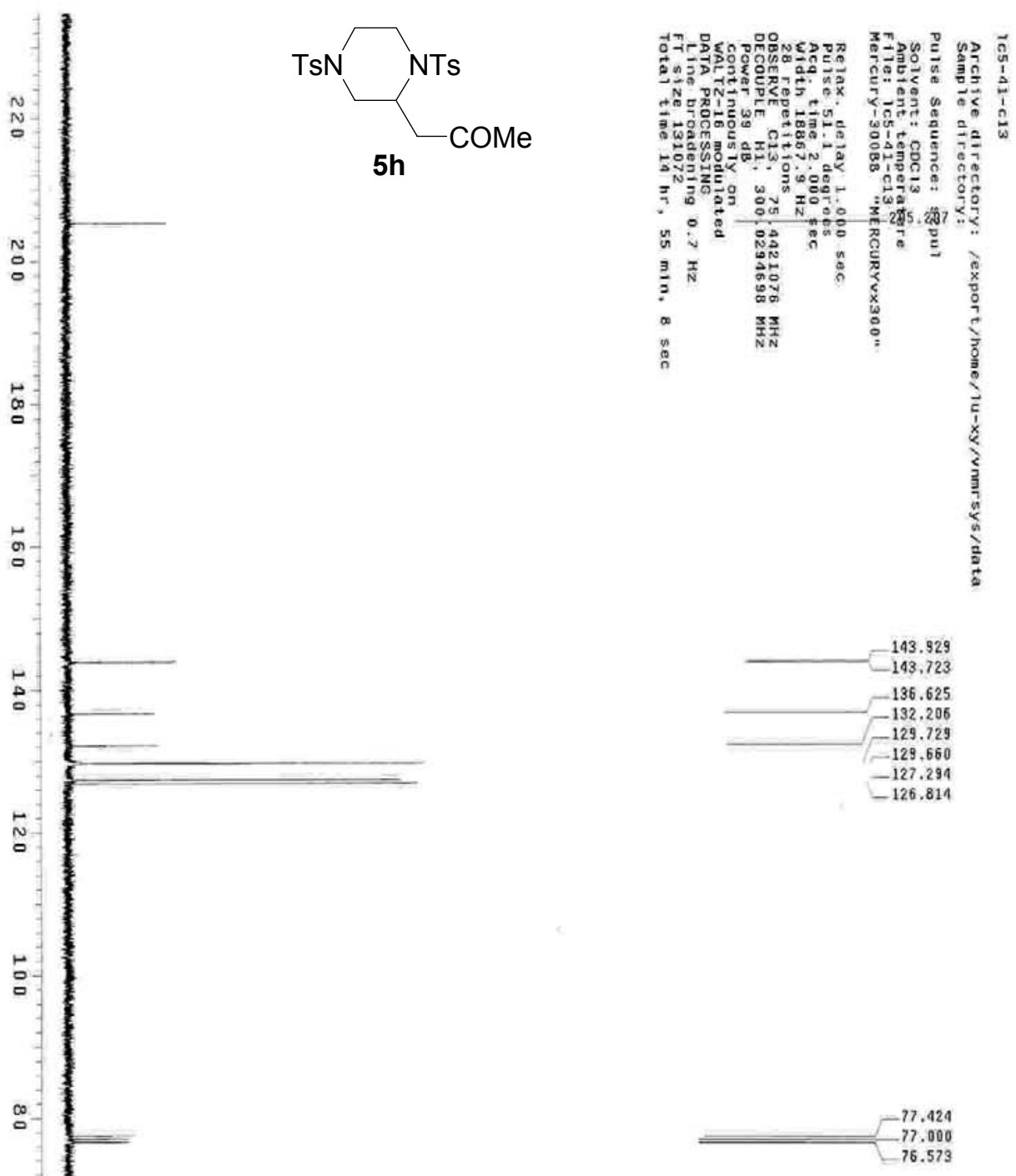

g.
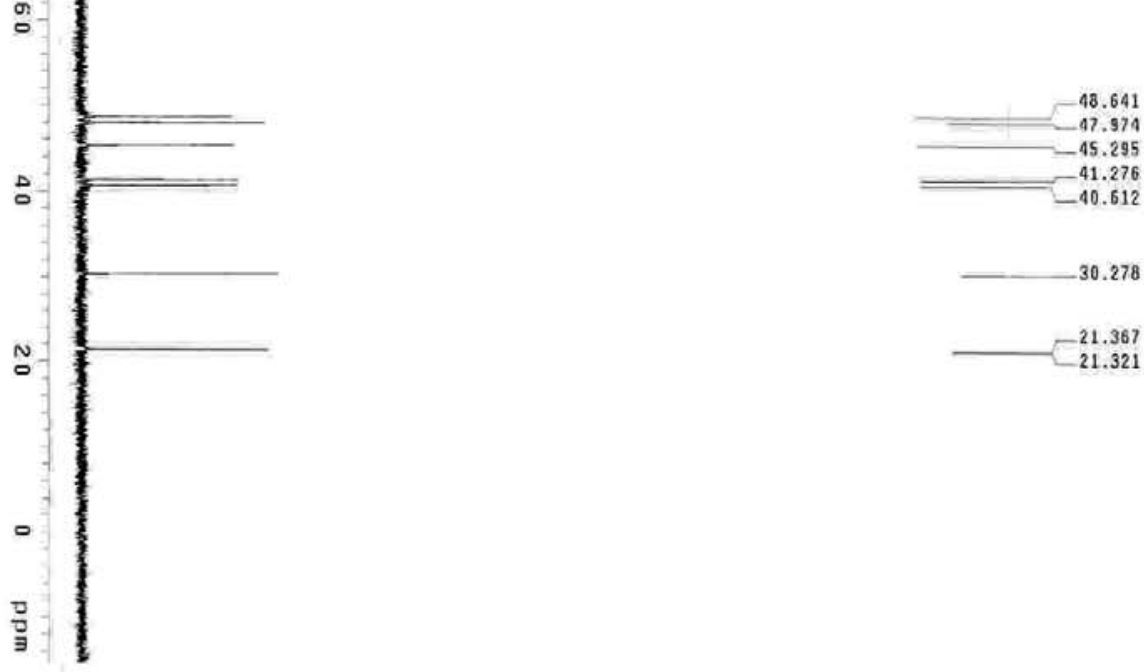

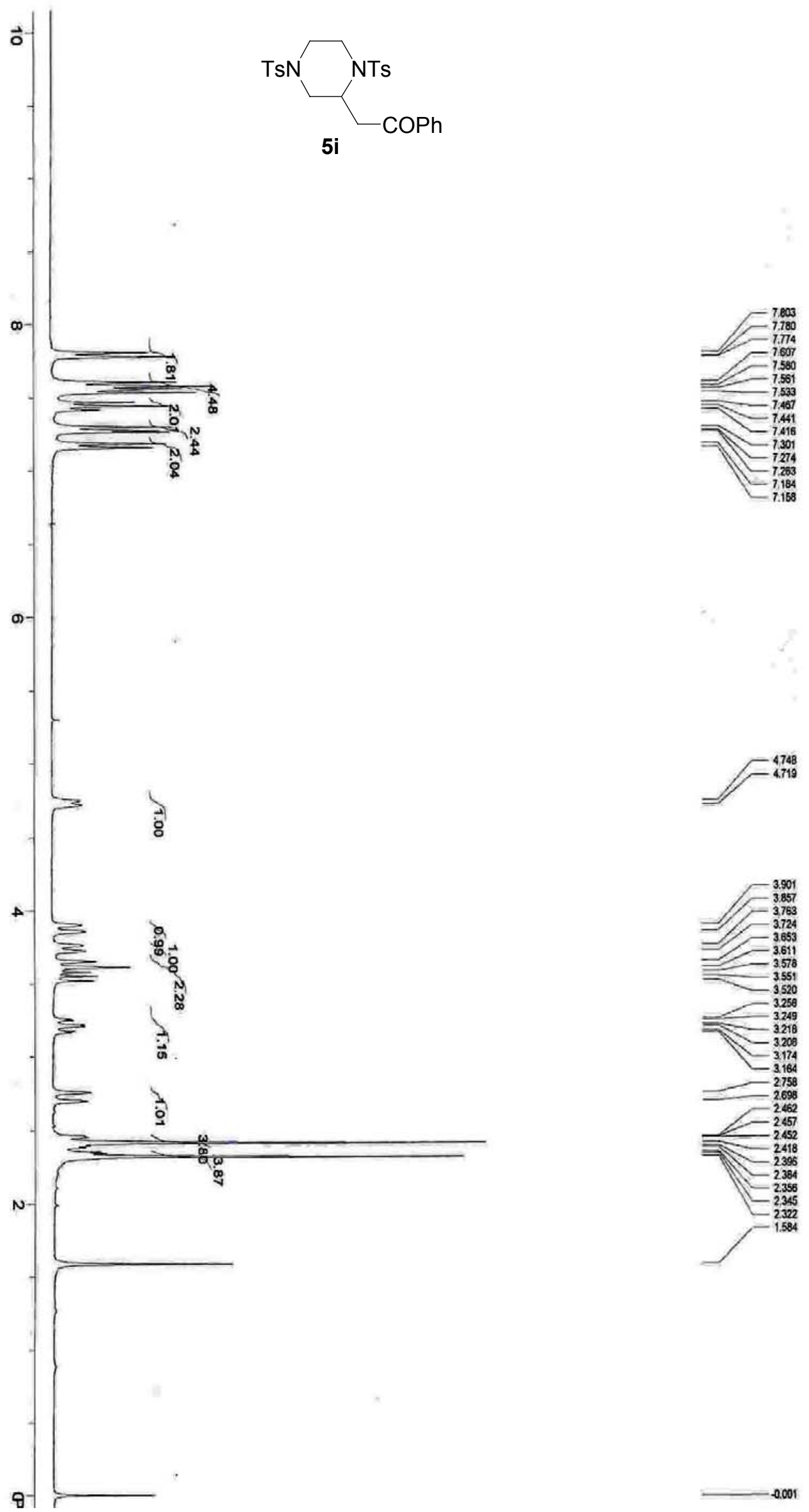

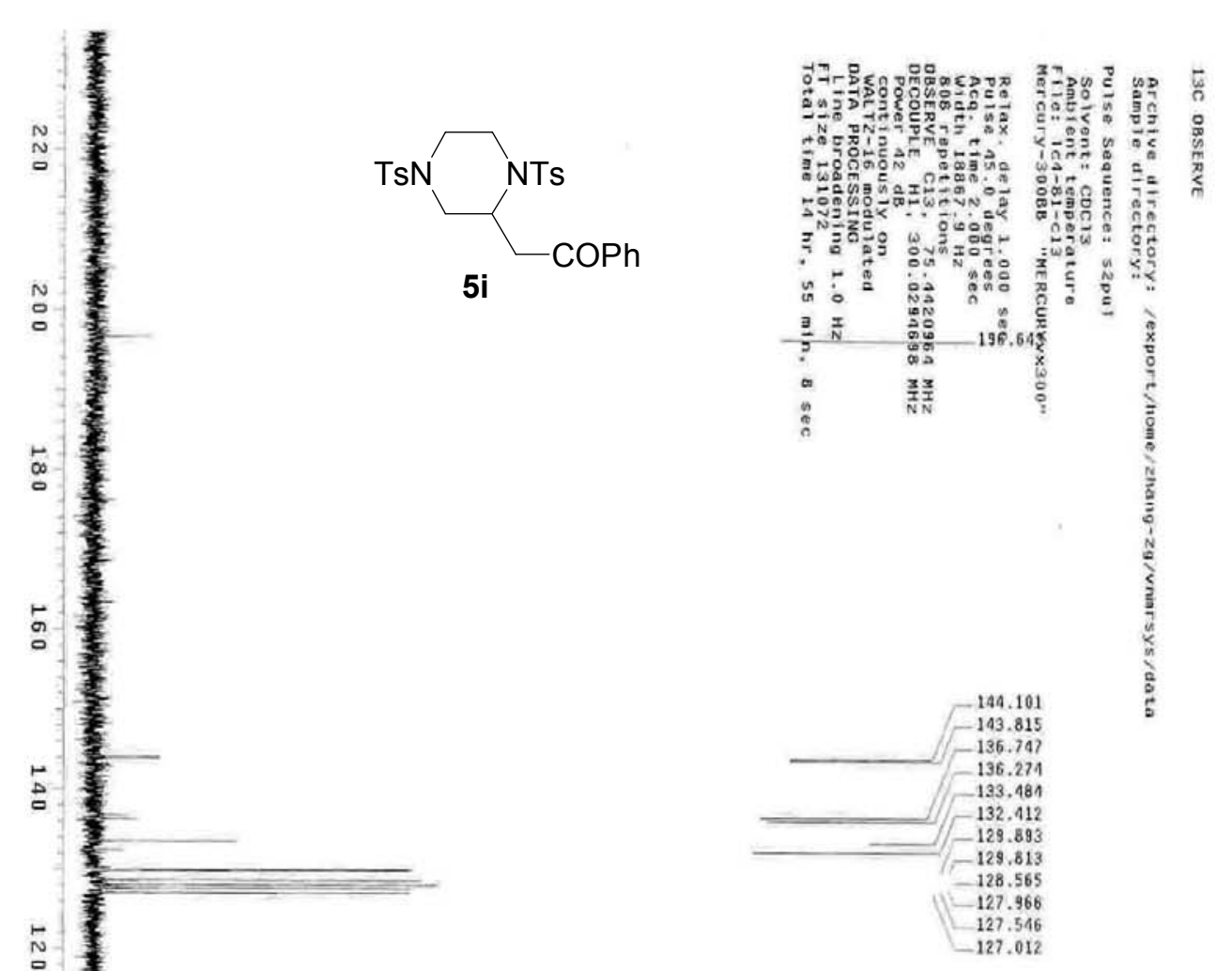

o

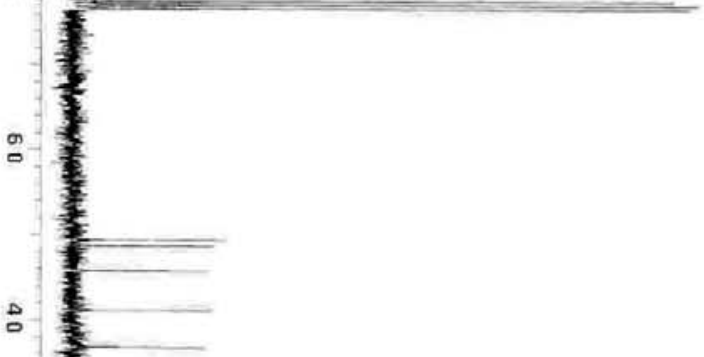

77.424

-77.000
-76.576

ง

-

.

:

,

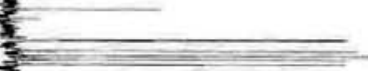

.

政
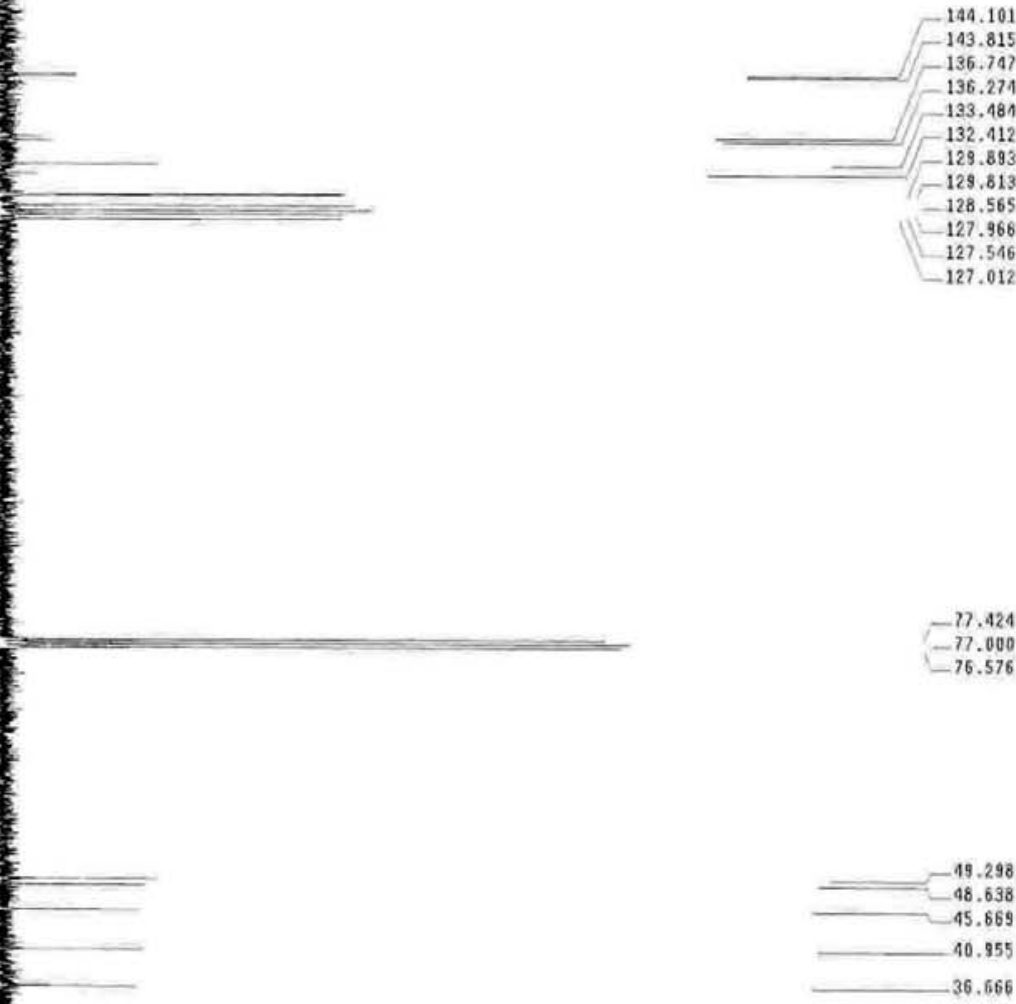

$-21.535$

$-21,432$

$=$

뭌 

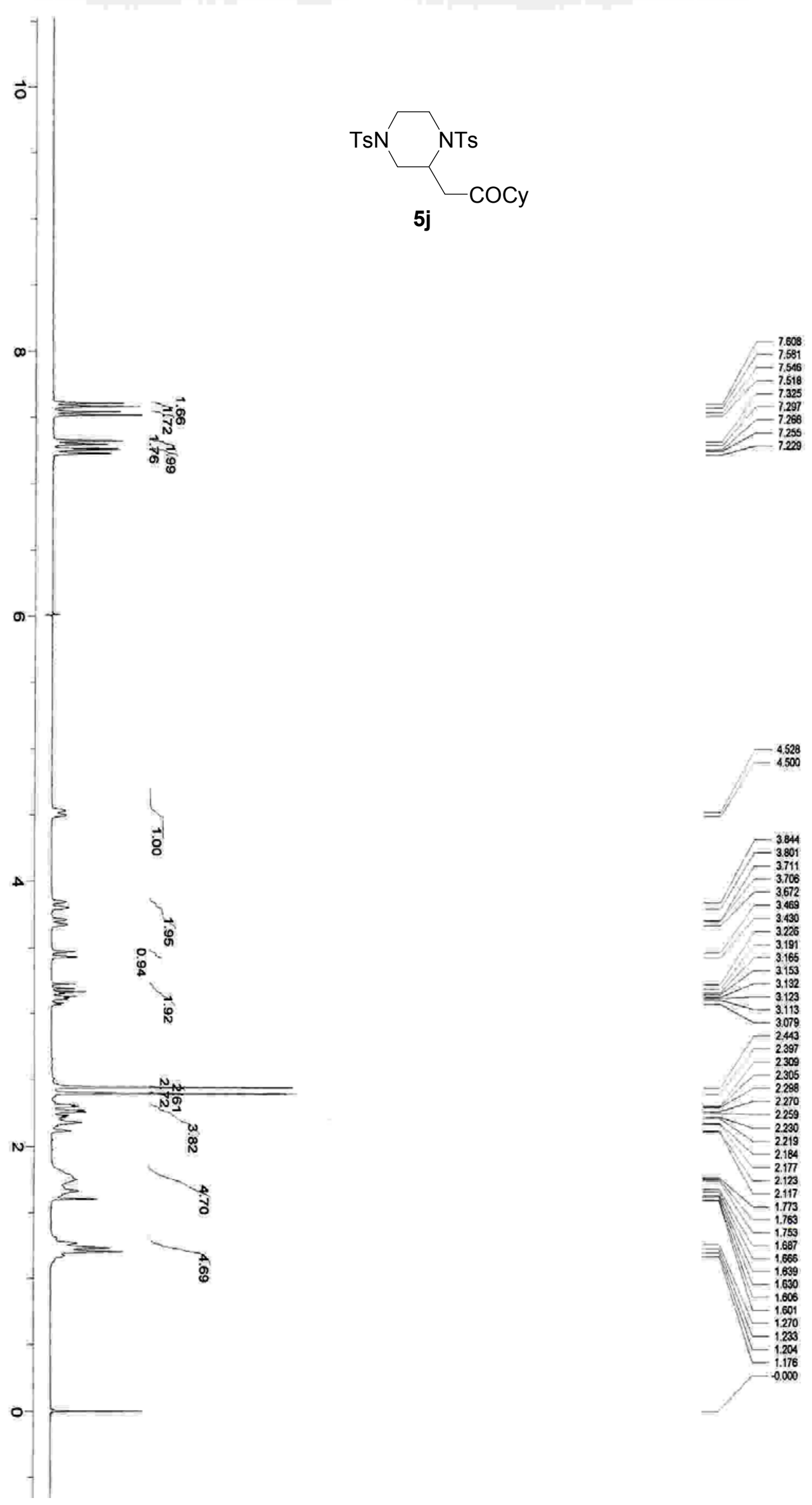

- $\quad 3.165$

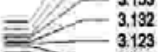

3.123
$-\quad 3.113$
-3079

-2443
-2.397
-2300

$\begin{array}{r}23095 \\ =\quad 2289 \\ -\quad 2270 \\ \hline\end{array}$

$\begin{array}{r}2239 \\ -230 \\ \hline\end{array}$

- $\begin{array}{r}2.114 \\ =2.177 \\ 2.123 \\ 2.17\end{array}$

- -2.117

-1.763
-1.753
-1.687

$=1.666$

I. $\begin{array}{r}-1.639 \\ -1.630\end{array}$

1.606
-1.601
-1.270
-1.230

-1.204
-1.176

$-0.000$ 


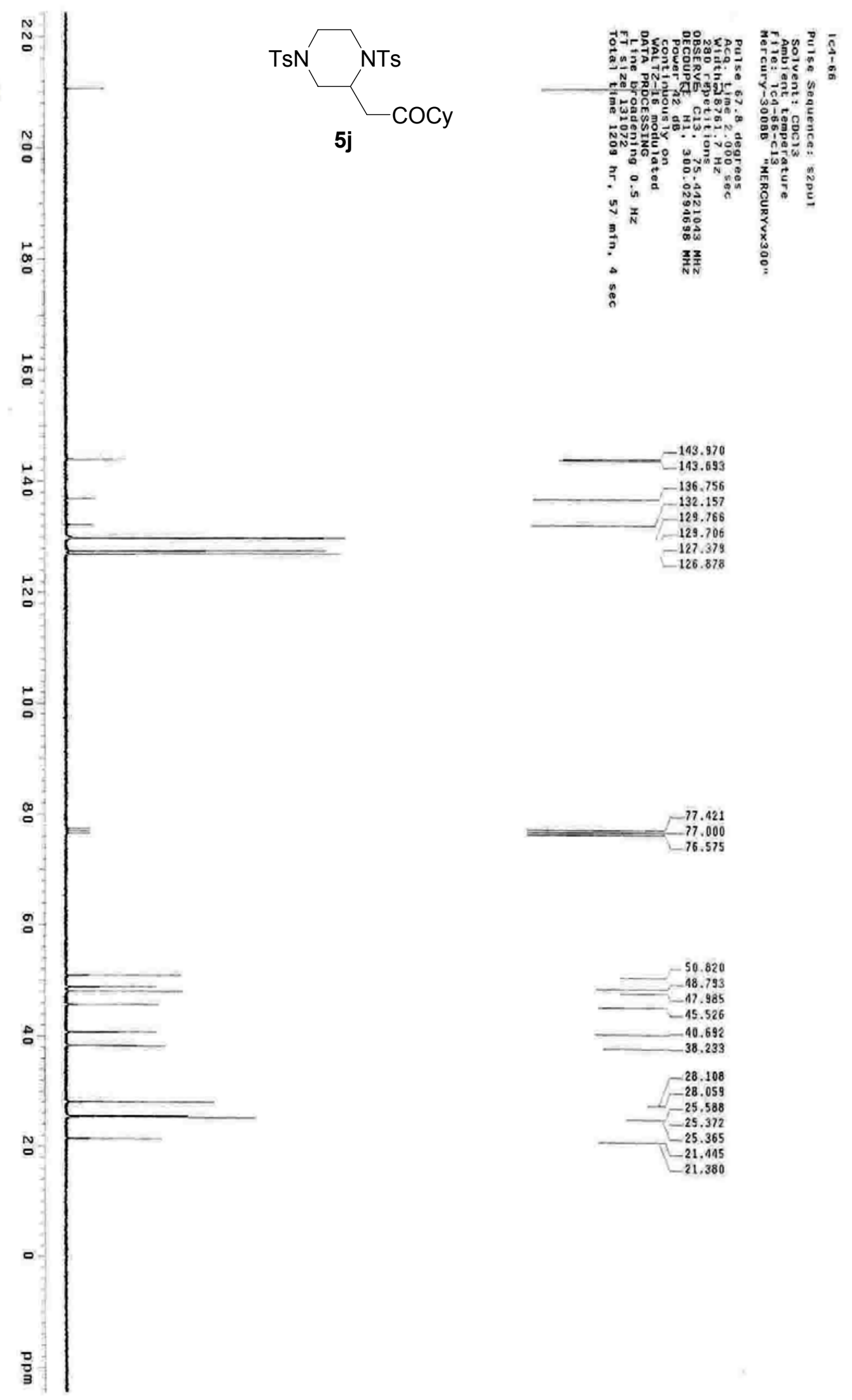



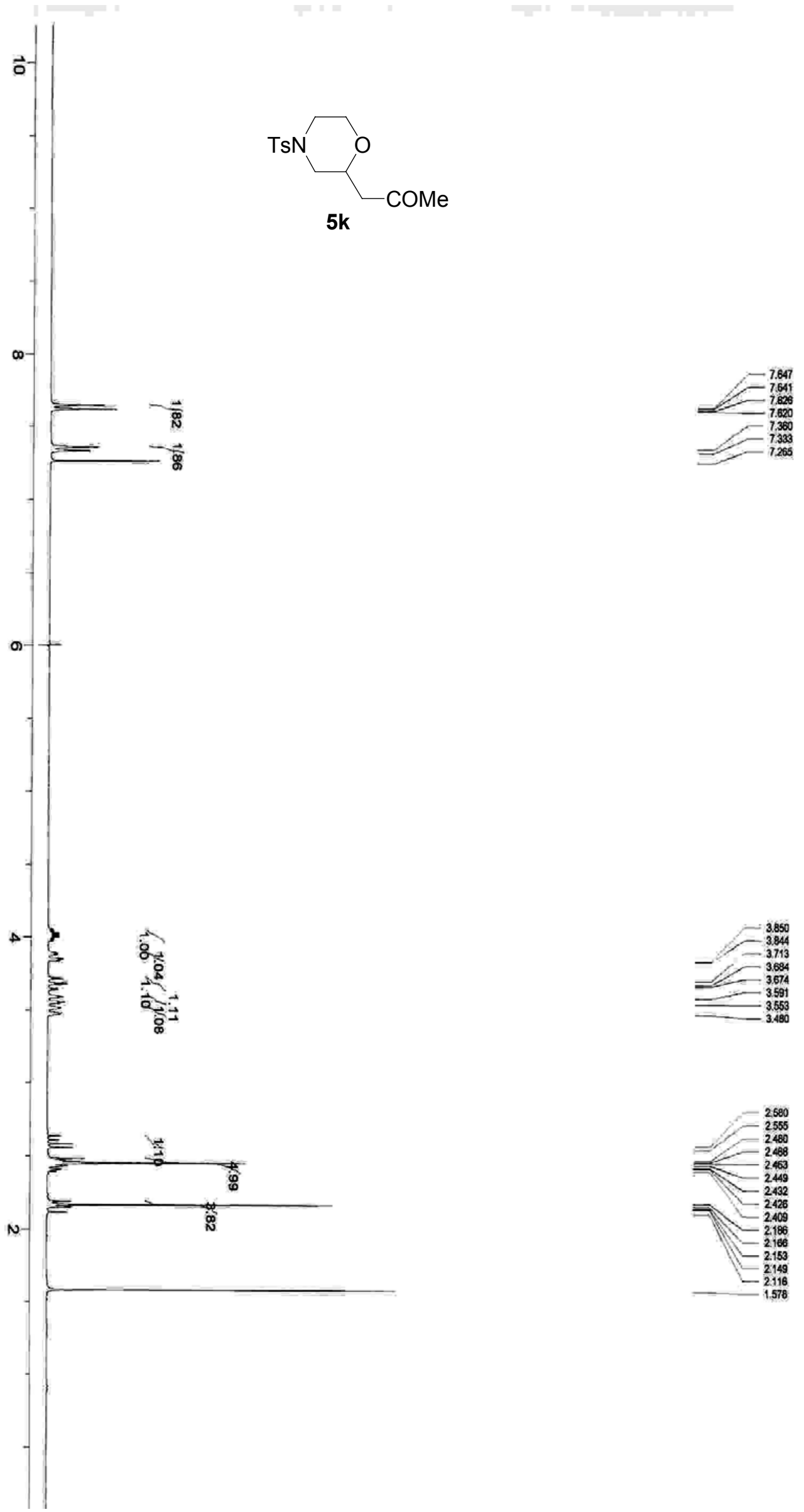


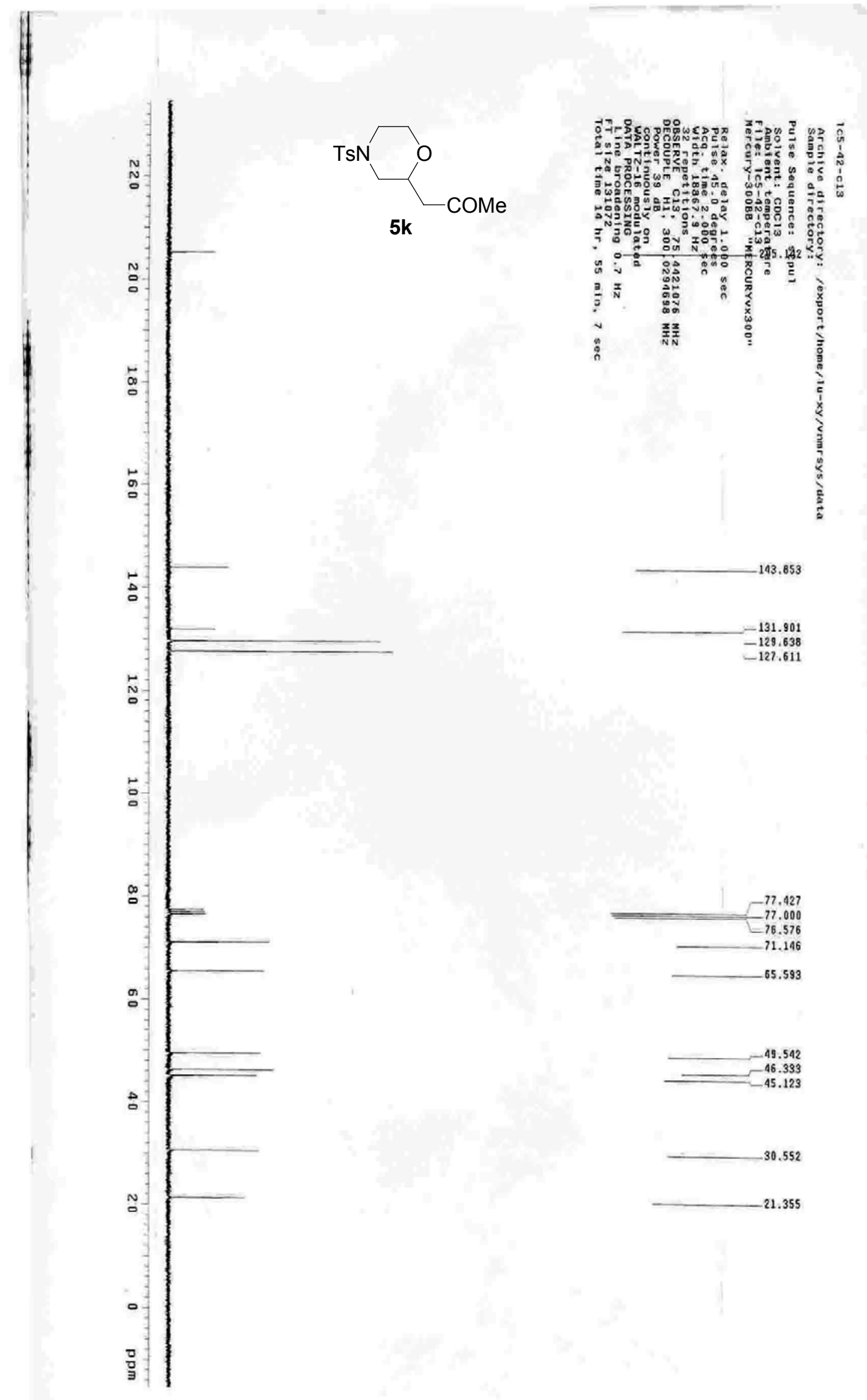




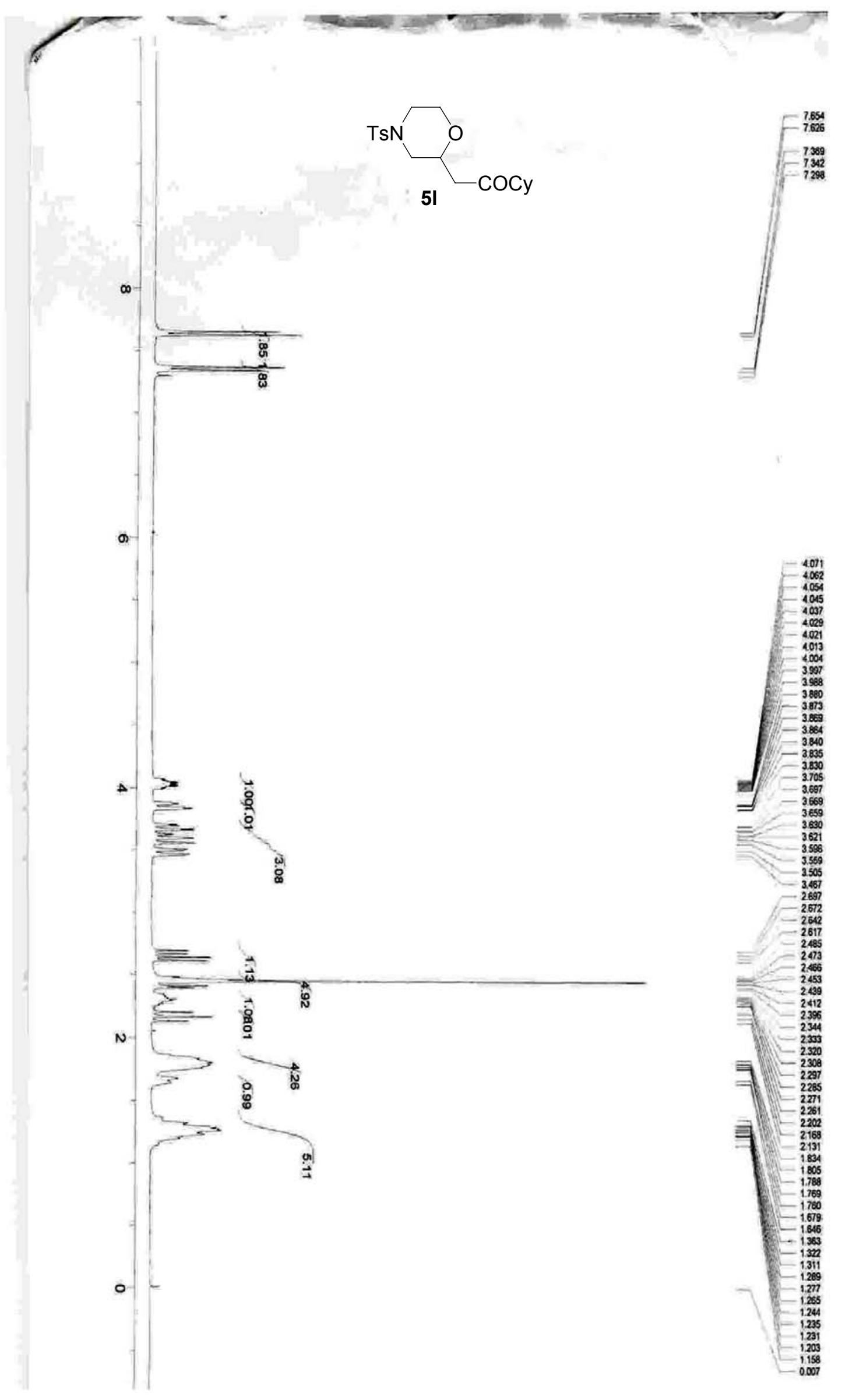



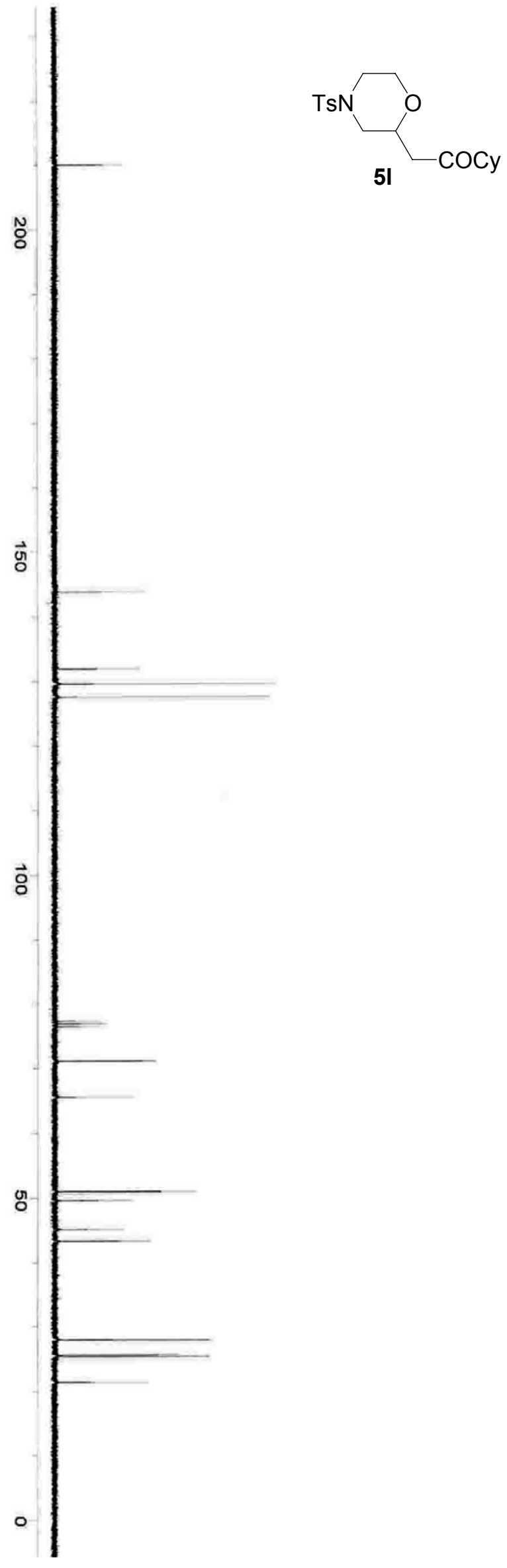

210.082

$=143767$

131992
129593
127508
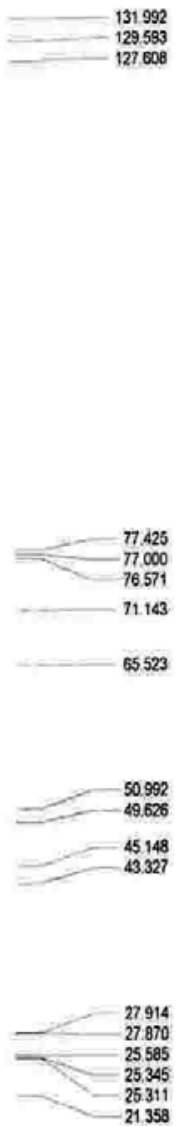


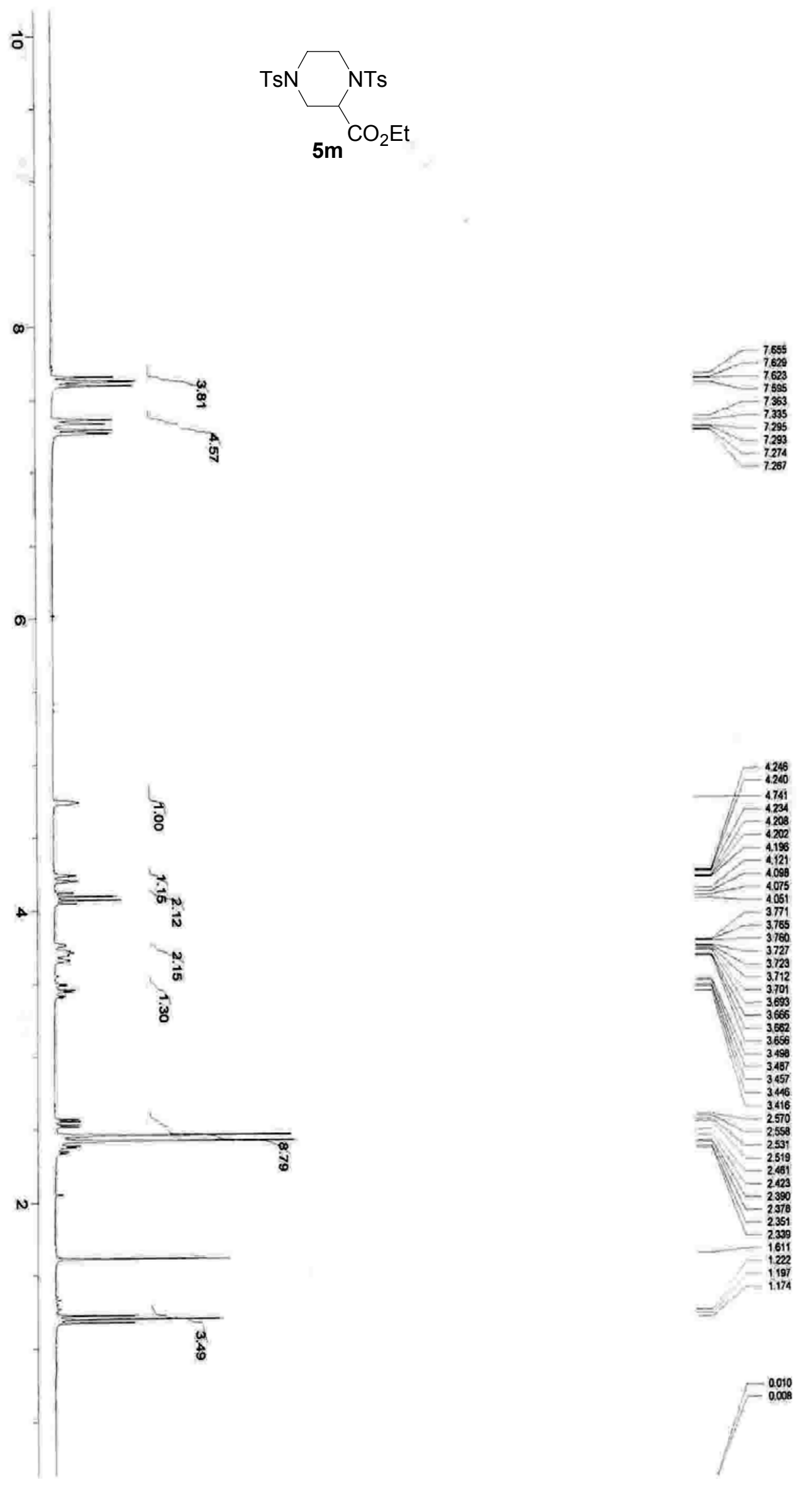



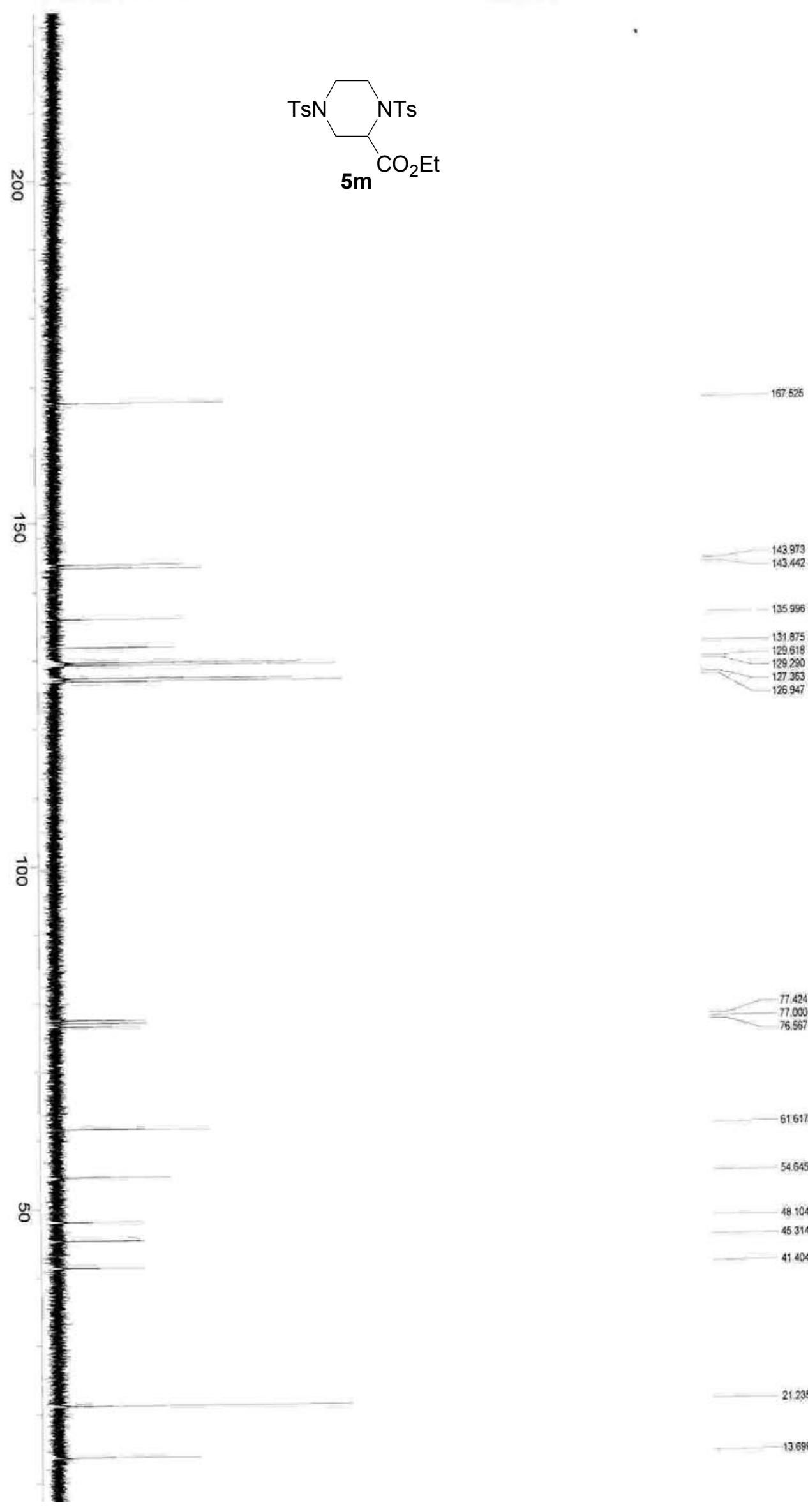

77.424
77.000

77.000
76.567

54645

48.104 $41 \mathrm{aOA}$ 

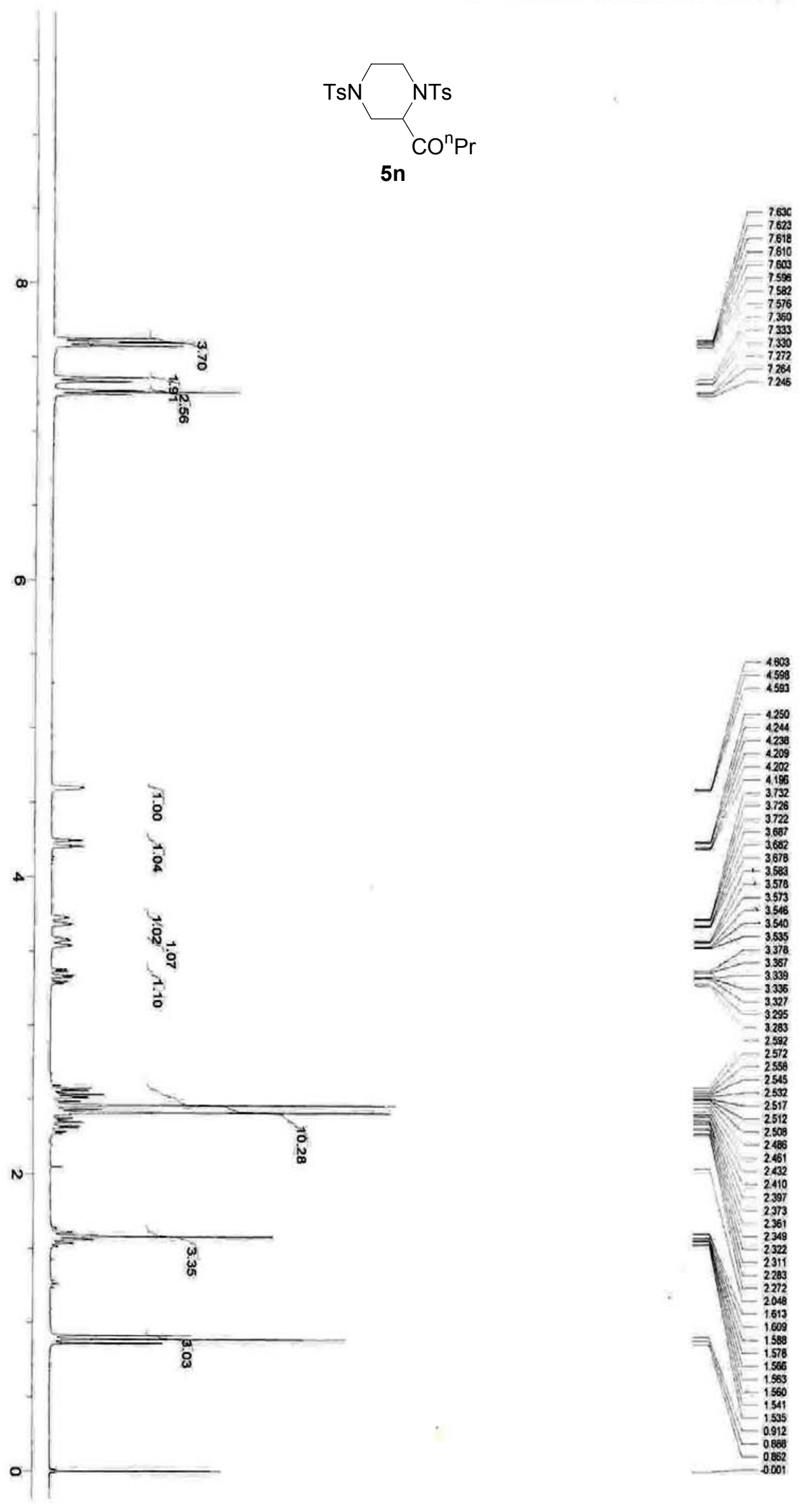

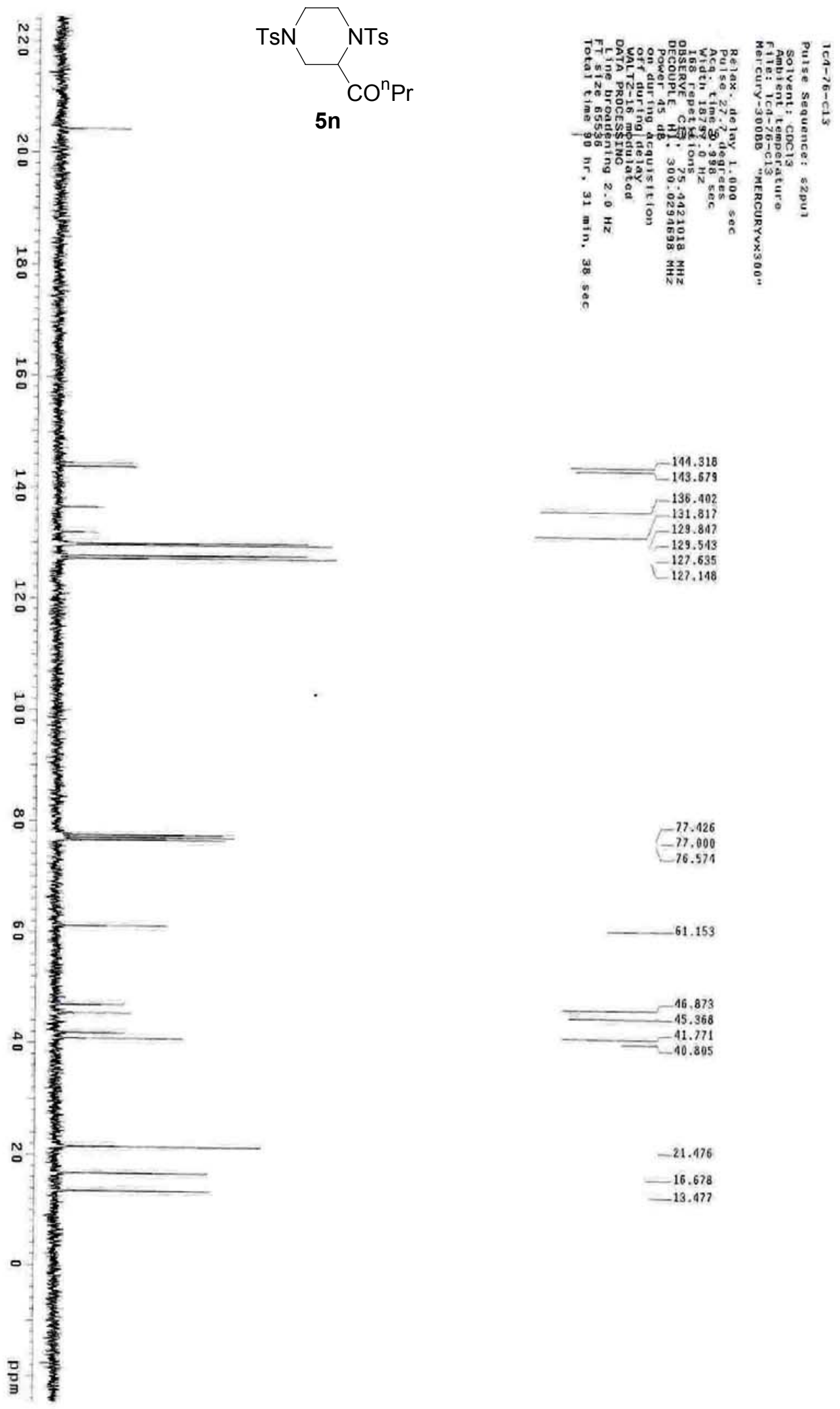

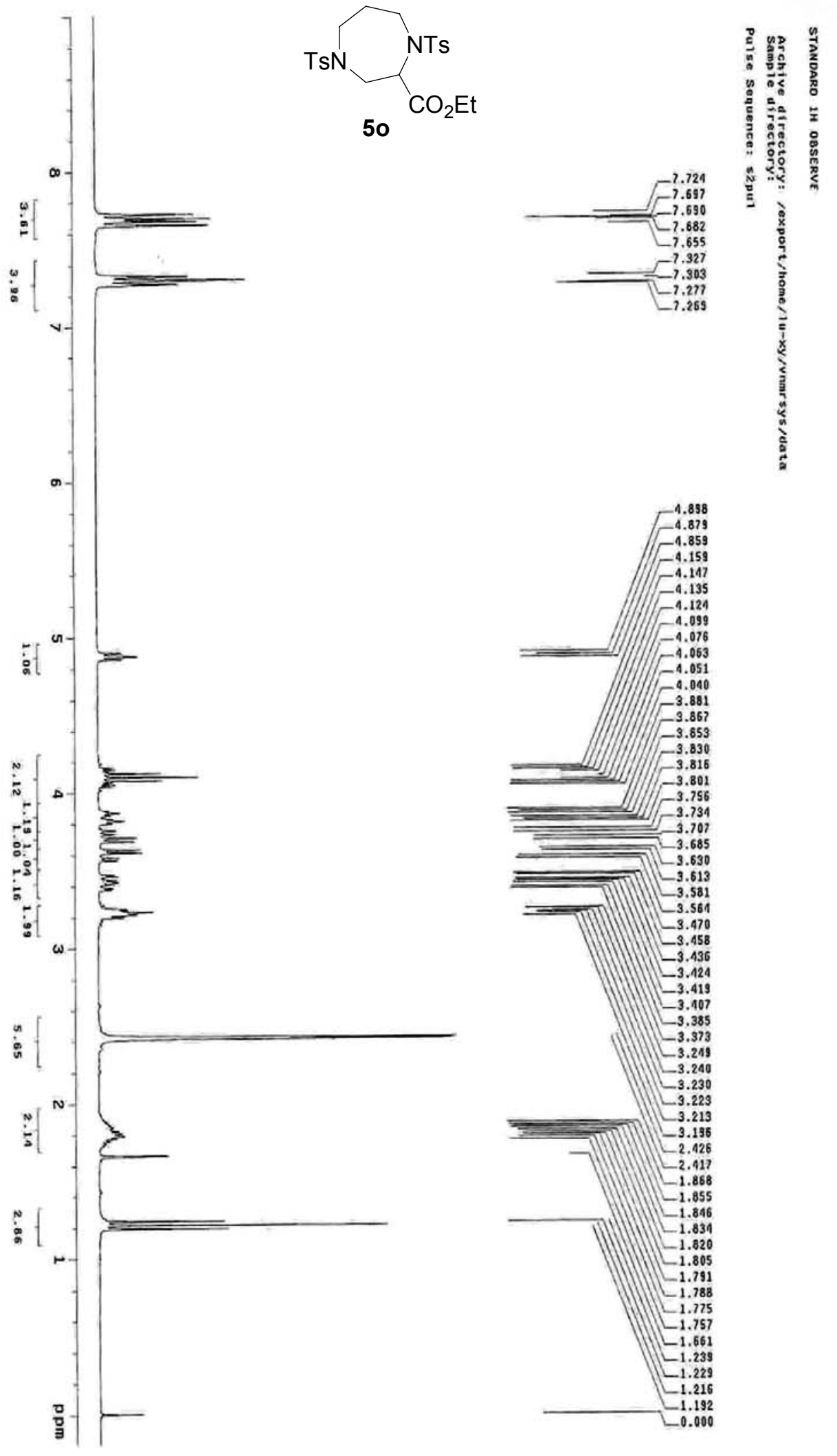

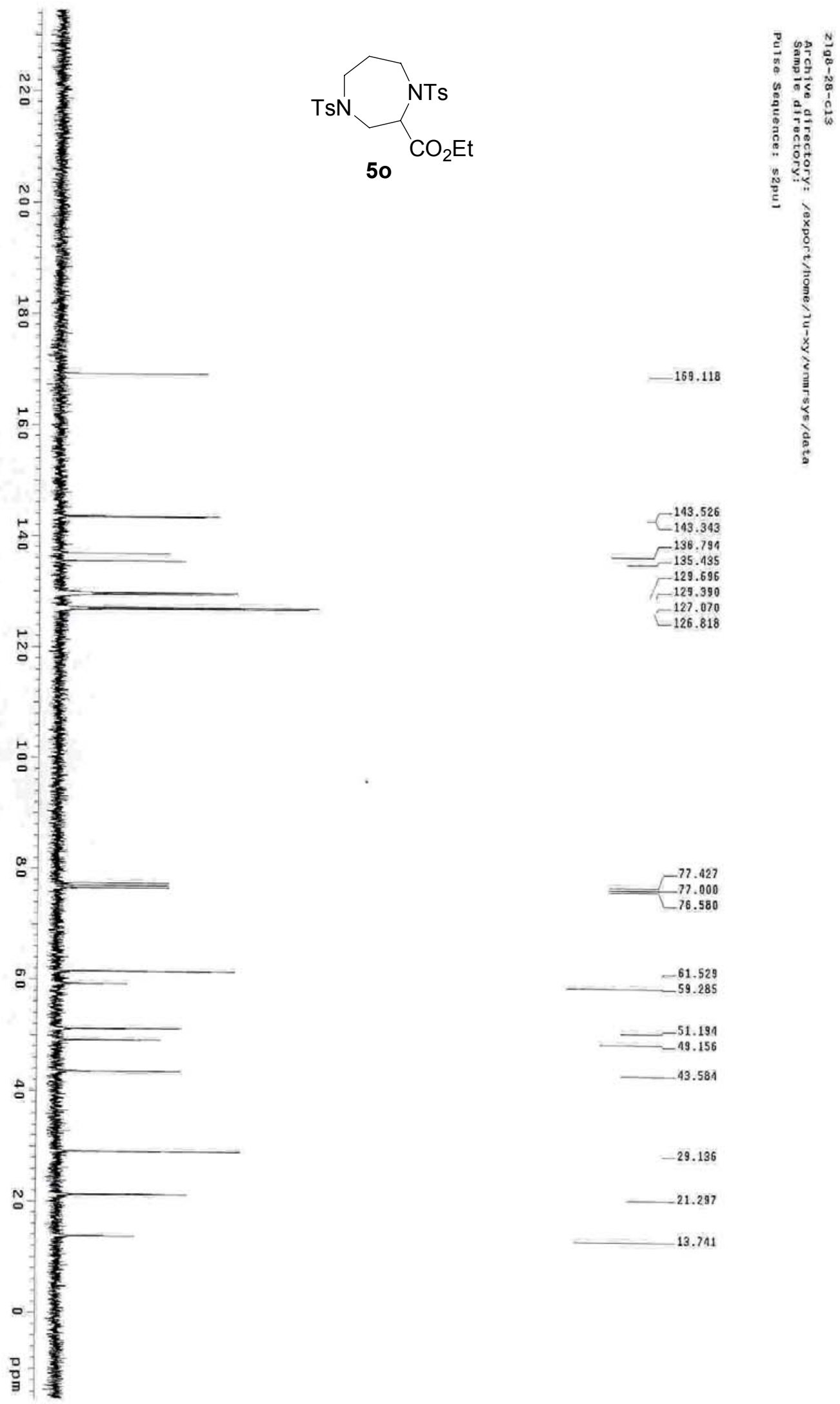

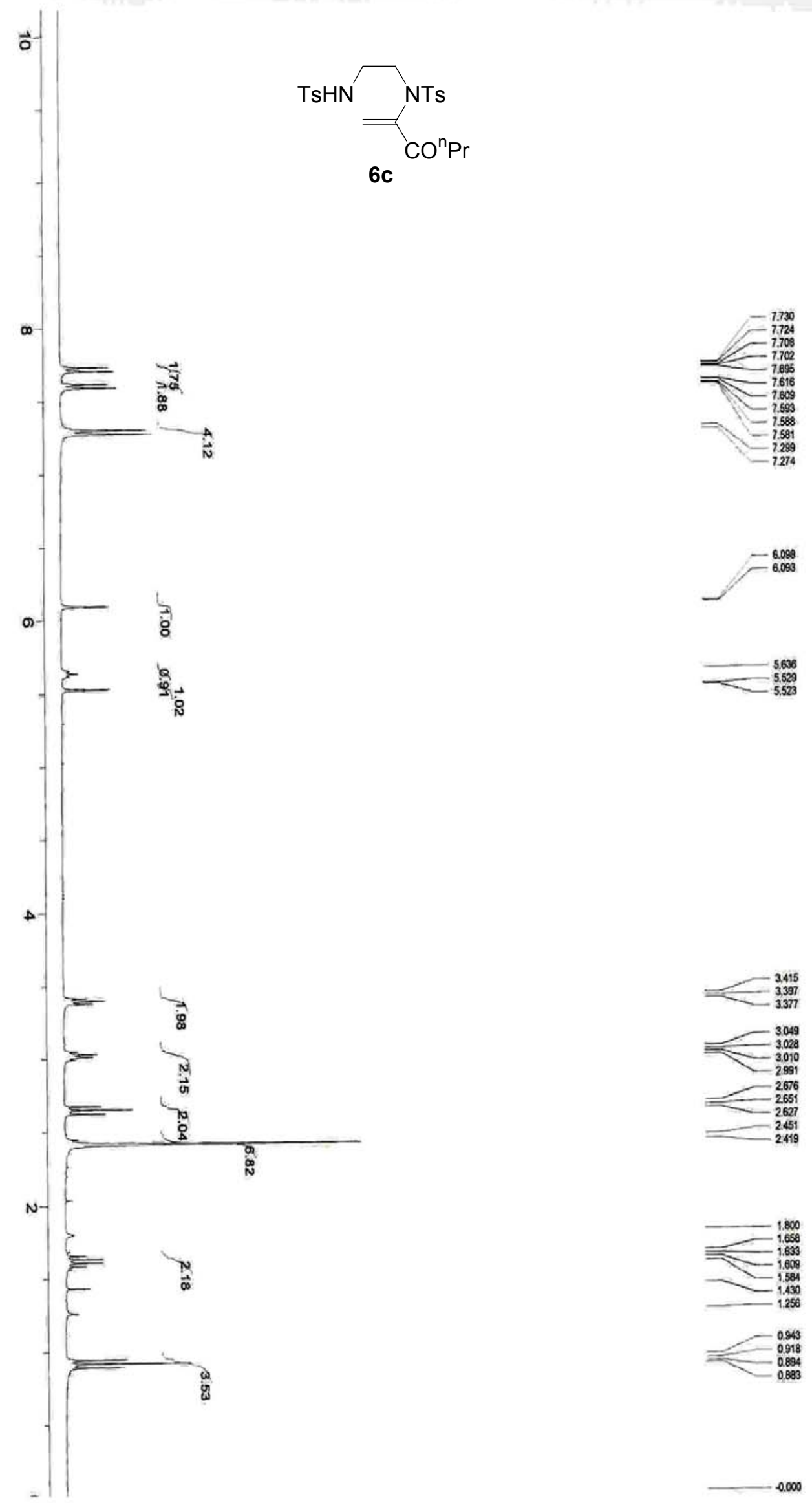

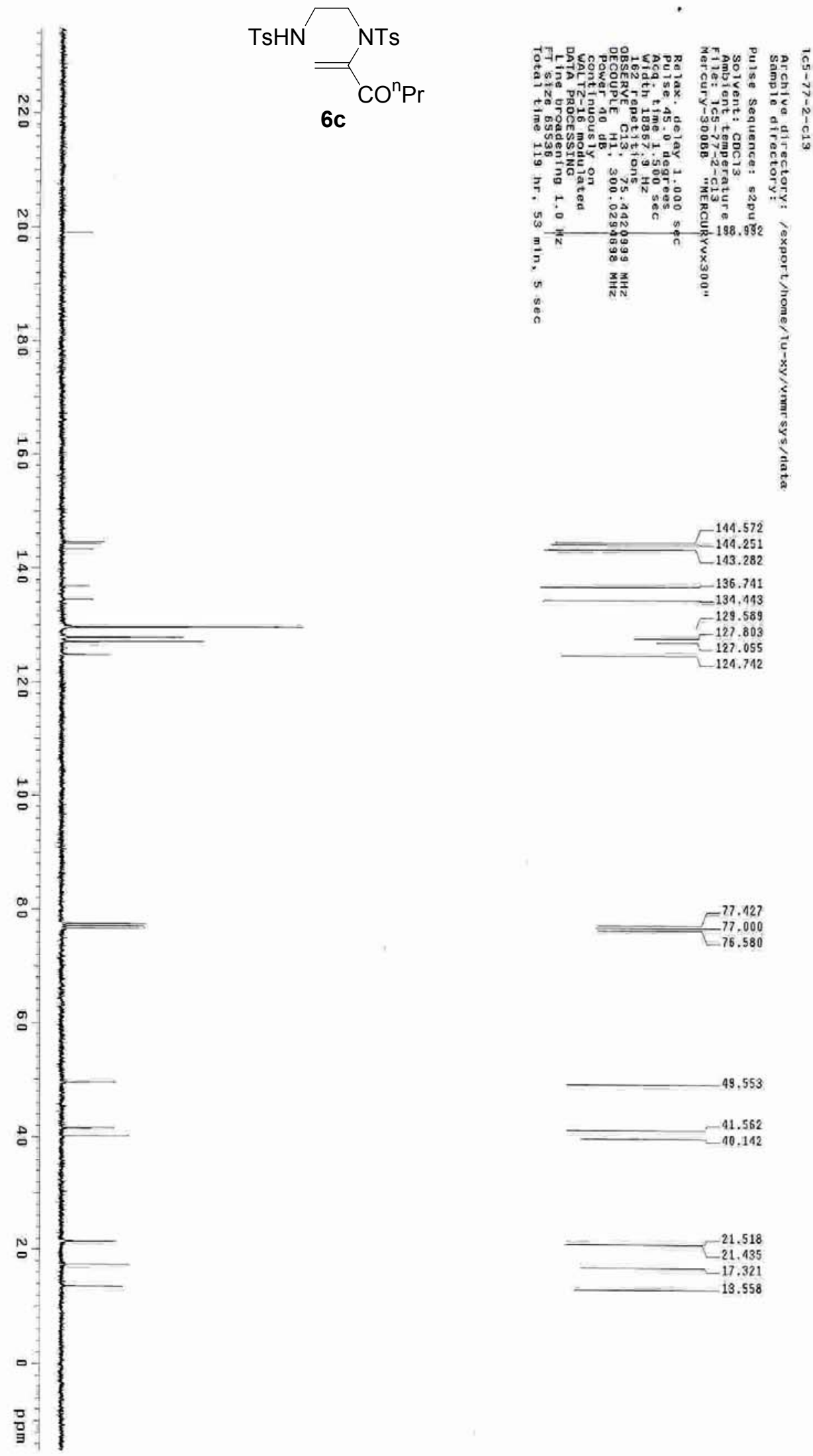